\title{
Prospects for new physics in rare decays, mixing and related CP violation at LHCb
}

Sebastian Bachmann on behalf of $\mathrm{LHCb}$ University of Heidelberg/CERN

Symposium on hadron collider physics Isola d'Elba, 21-25 May 2007 


\section{Motivation}

A lot of precise measurements are available from B-factories and Tevatron to test the CKM picture of flavour structure and $\mathrm{CP}$ violation.
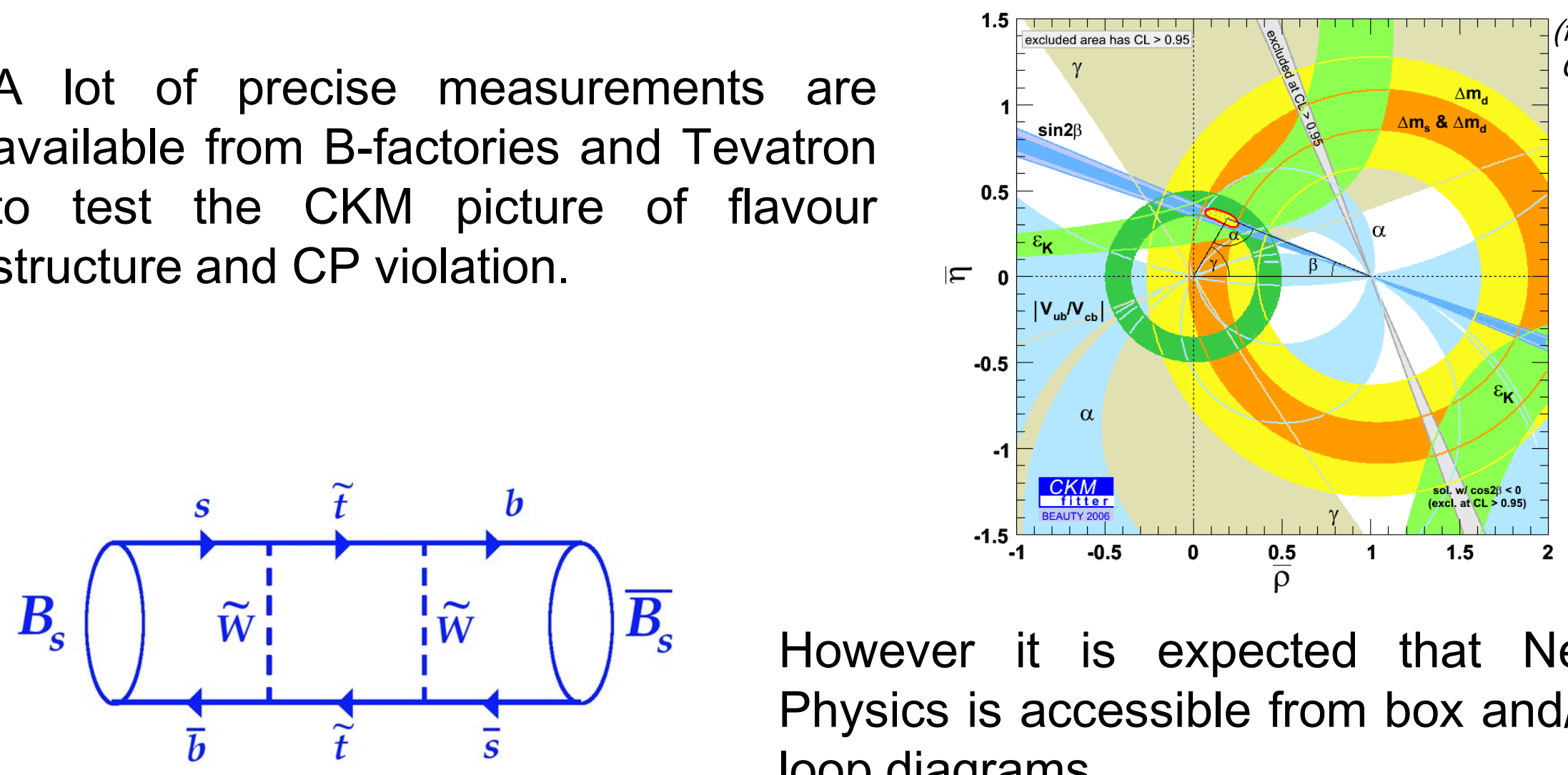

(from

CKM-fitter)

However it is expected that New Physics is accessible from box and/or loop diagrams.

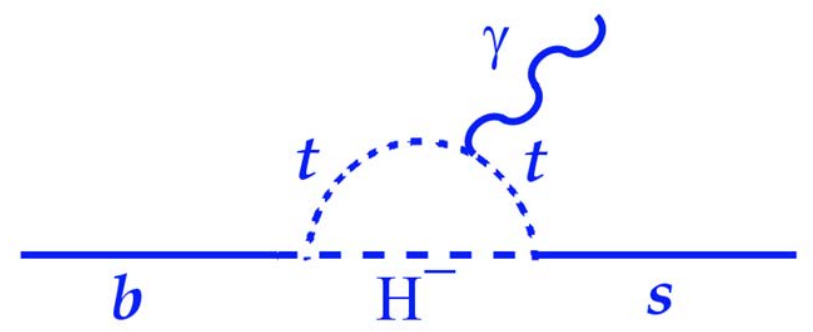

LHCb aims to find New Physics contributions in these processes. 


\section{What do we get from LHC?}

$>$ bb cross section: $500 \mu b+$

LHCb luminosity $\sim 2-5 \cdot 10^{32} \mathrm{~cm}^{-2} \mathrm{~s}^{-1}$

(avoid Pile-Up!)

$G$ b-production rate $\sim 100 \mathrm{kHz}$

\section{One year of nominal data taking} corresponds to $2 \mathrm{fb}^{-1}$
Inelastic pp collisions/crossing: For LHCb mainly single interactions

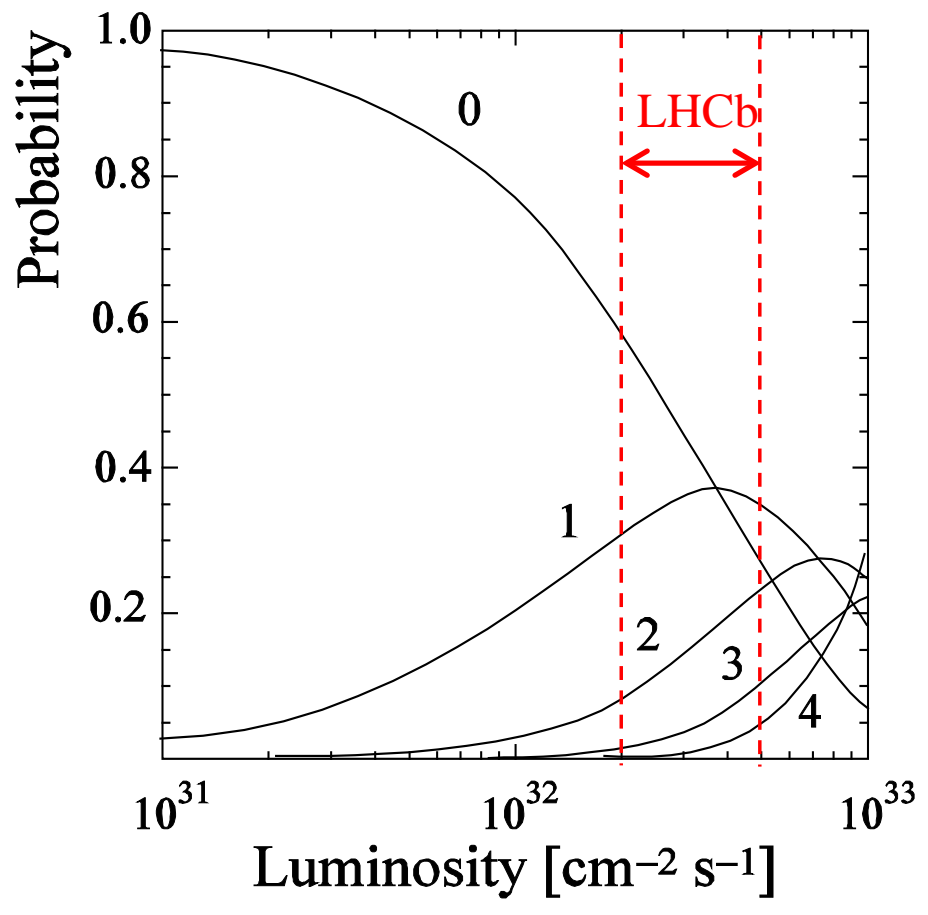




\section{A signal event: $B_{S} \rightarrow D_{S}^{-}\left(K^{+} K^{-} \pi^{+}\right) K^{+}$}

\section{Signal side $\quad \mathrm{K}^{+}$}

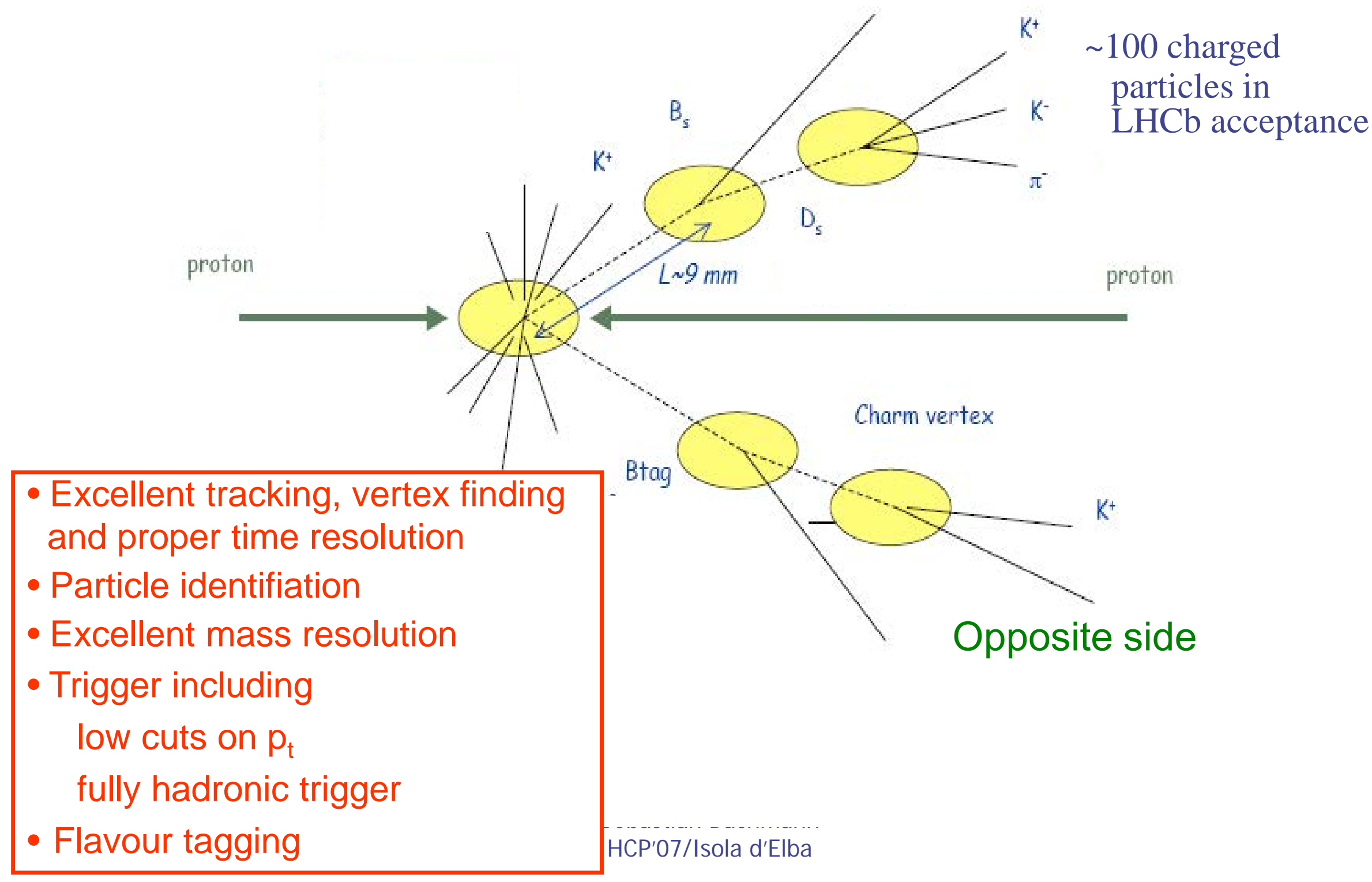




\section{LHCb detector}

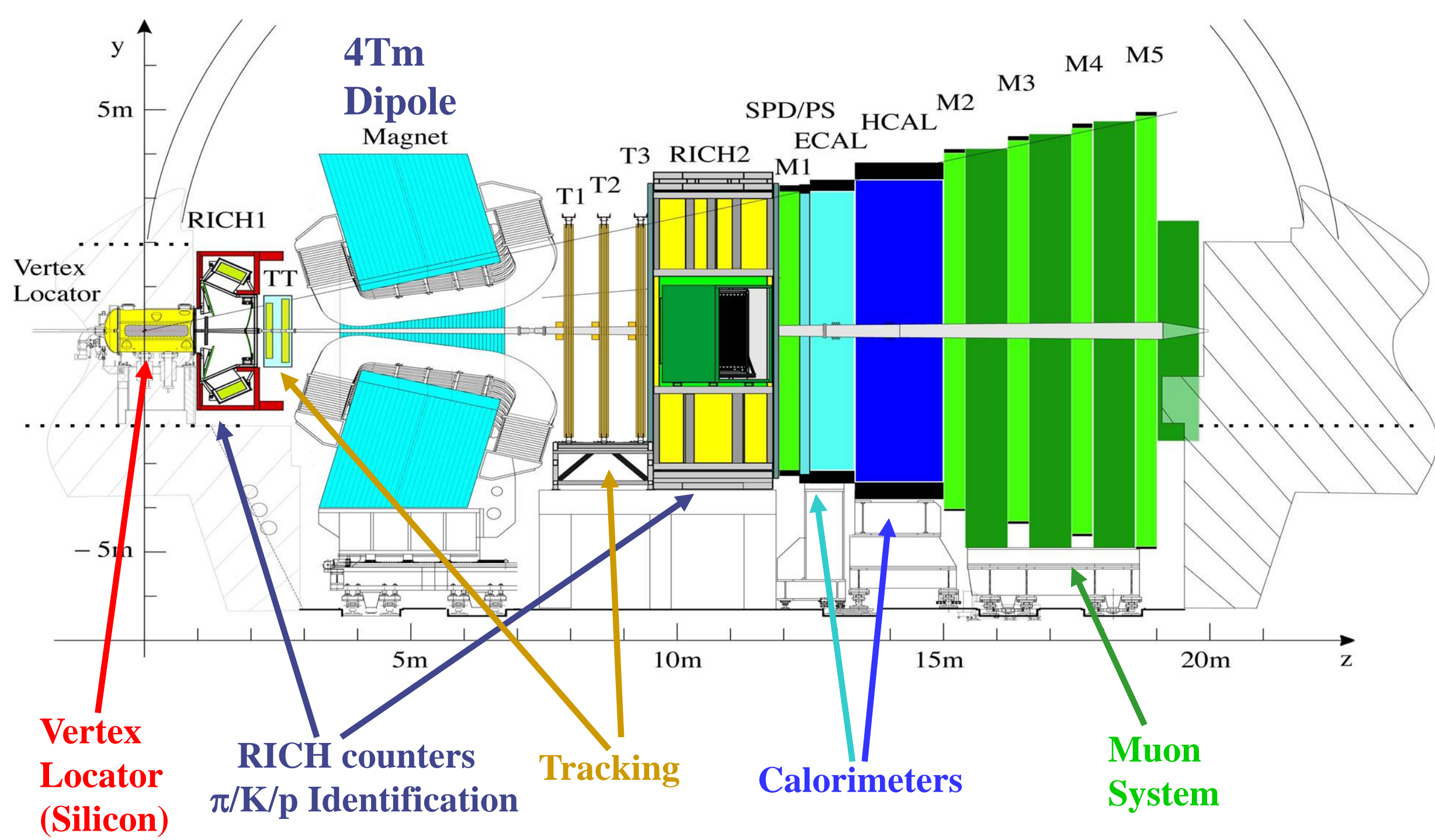




\section{LHCb detector in place}

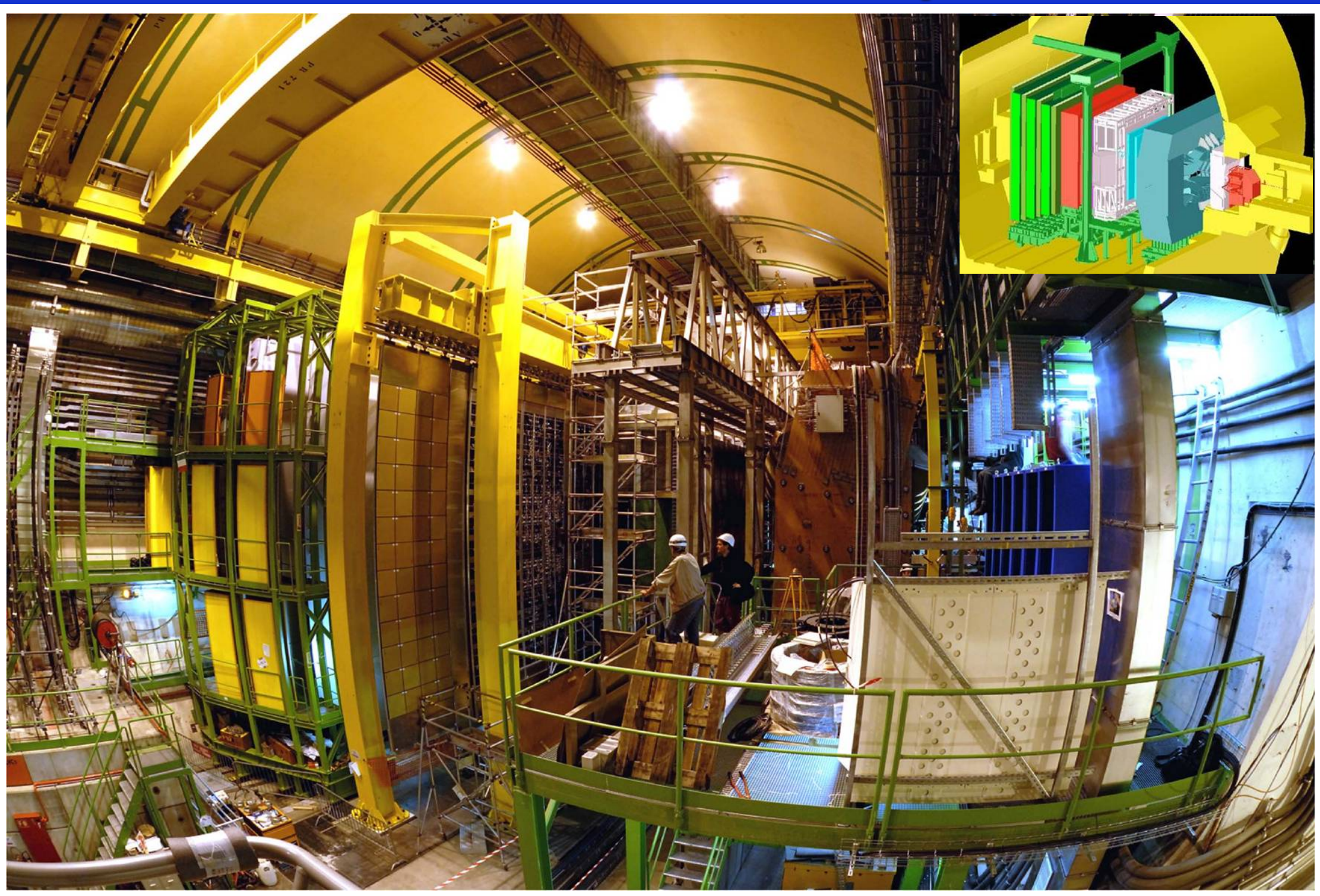




\section{LHCb performance:}

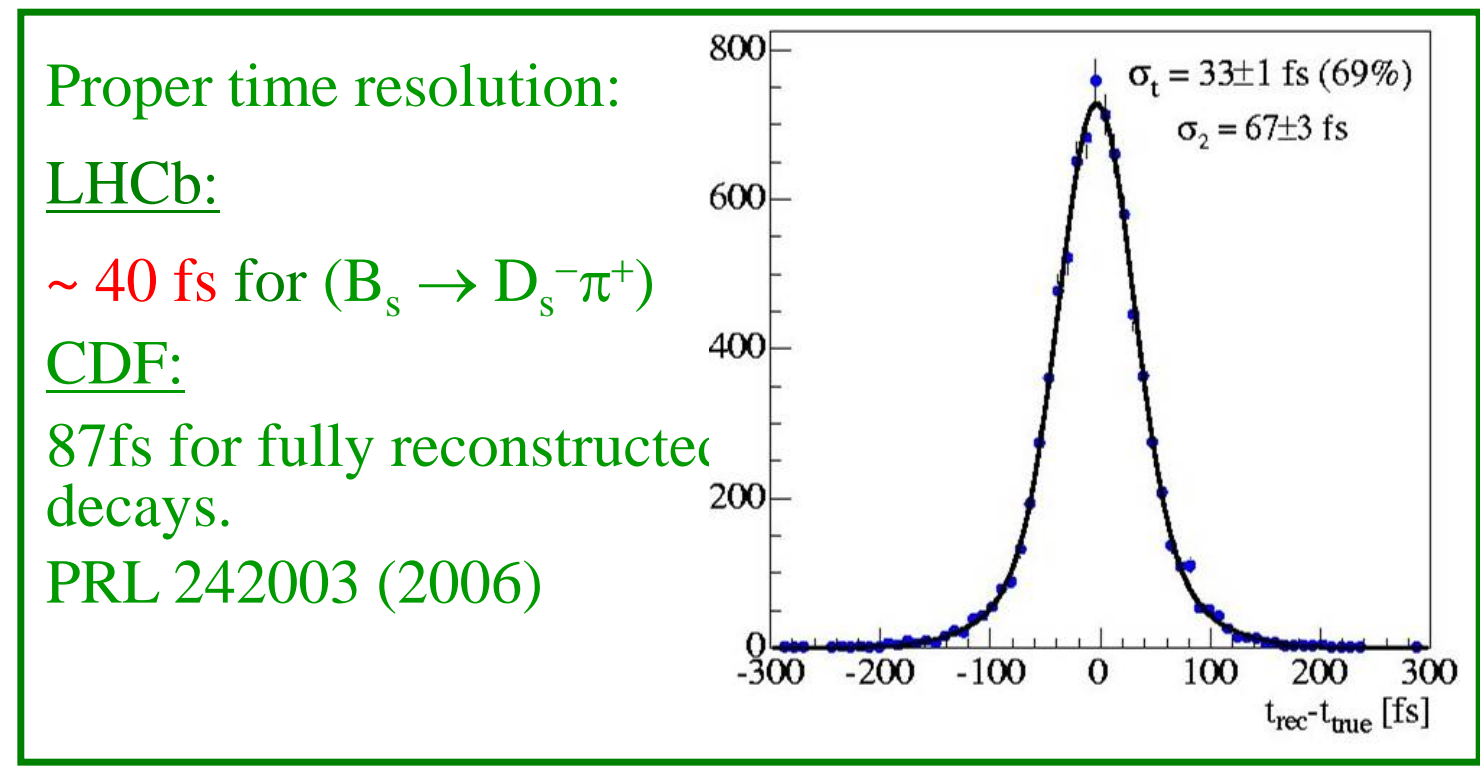

$\pi-\mathrm{K}$ separation:

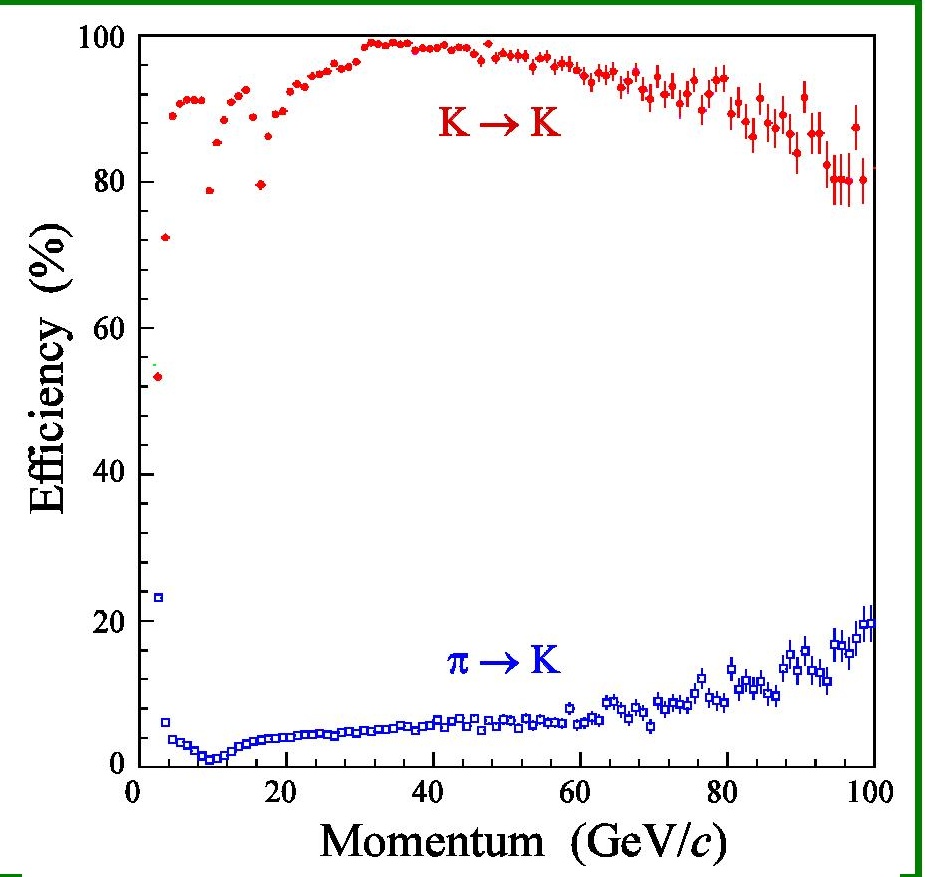

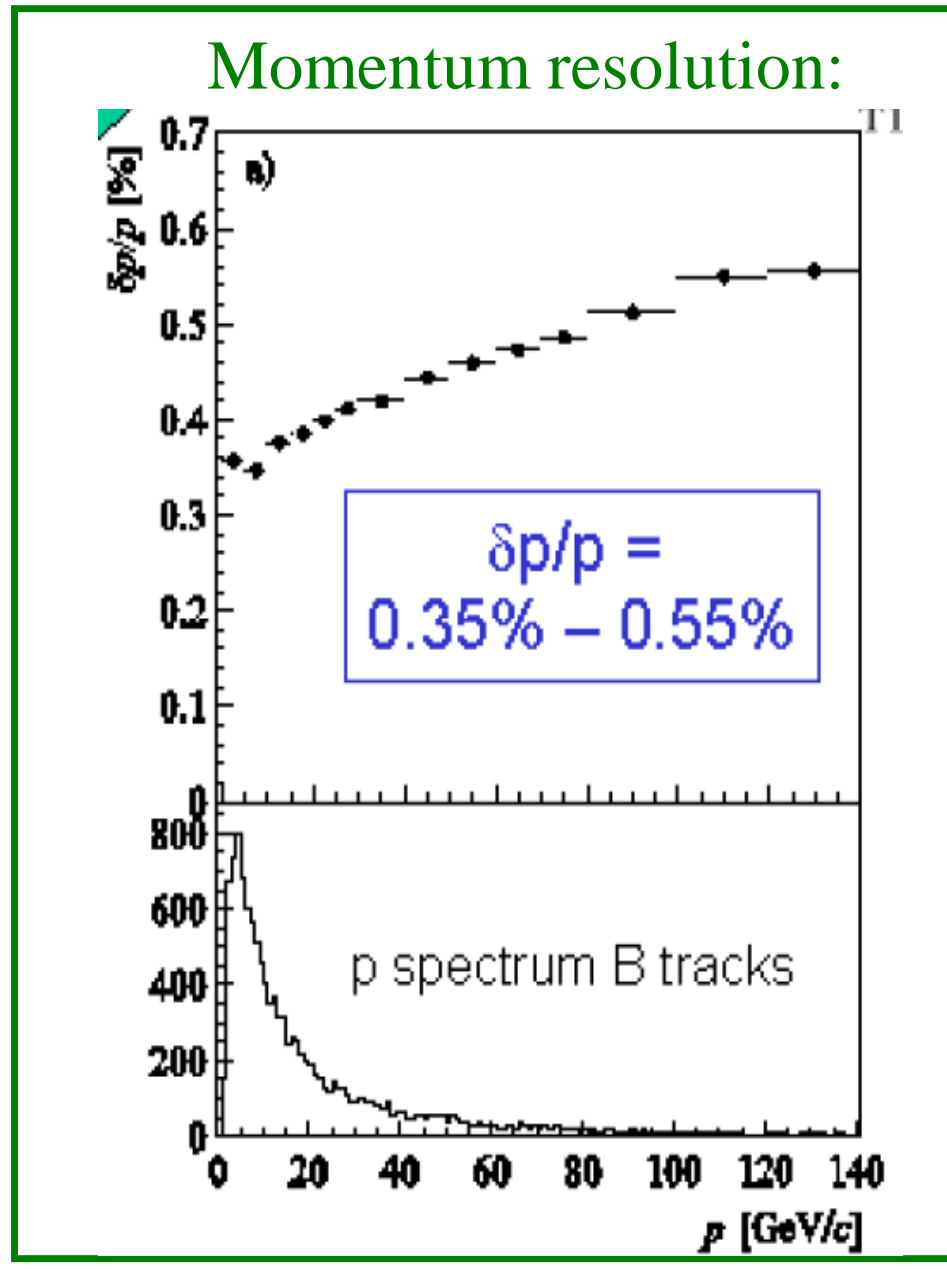

7 


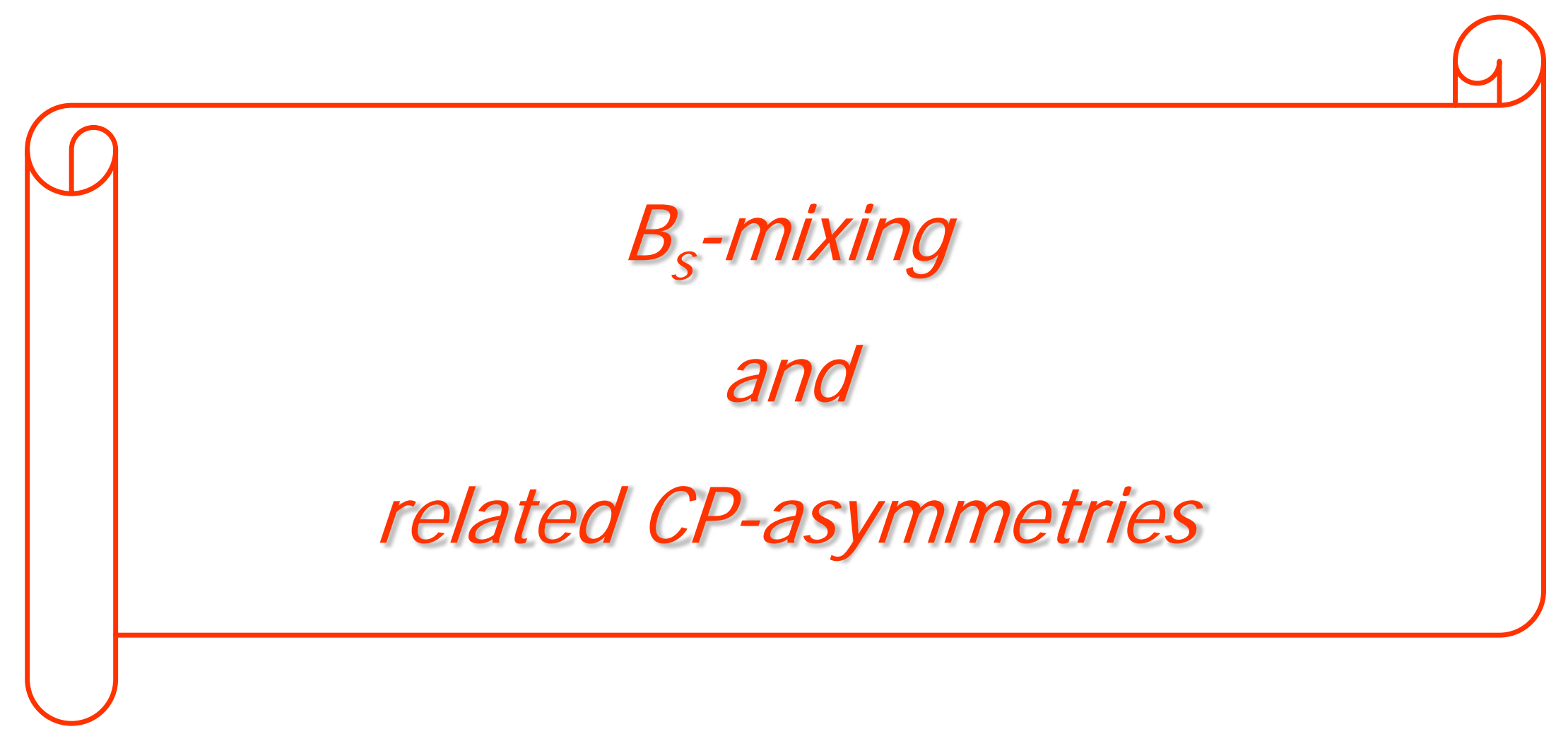




\section{NP from mixing and CP-asymmetries}

$\underline{\mathrm{B}}_{\mathrm{s}} \rightarrow \mathrm{D}_{\mathrm{s}} \pi^{+}: \quad>\mathrm{CDF}: \Delta \mathrm{m}_{\mathrm{s}}=\left(17.77 \pm 0.1^{\text {stat }} \pm 0.07^{\text {syst }}\right) \mathrm{ps}^{-1}$

Precise measurement of $\Delta \mathrm{m}_{\mathrm{s}}>$ LHCb: Observation expected after few month using $\mathrm{B}_{\mathrm{s}} \rightarrow \mathrm{D}_{\mathrm{s}}^{+} \pi^{-}$.

$\underline{\mathrm{B}}_{\mathrm{s}} \rightarrow \mathrm{J} / \psi \Phi:$

Extract $\Phi_{\mathrm{s}}$ and $\Delta \Gamma_{\mathrm{s}}$ in golden mode $\mathrm{B}_{\mathrm{s}} \rightarrow \mathrm{J} / \psi \Phi$.

$(\mathrm{NP} \rightarrow$ contribution to box diagram)

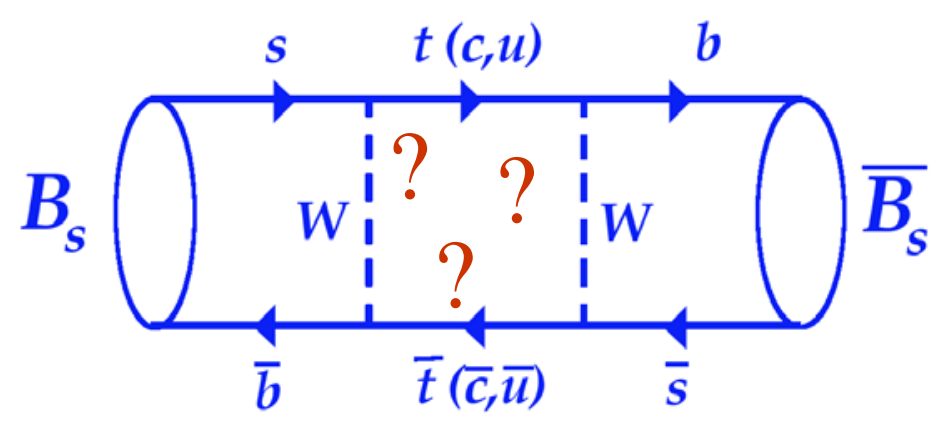

$\underline{\mathrm{B}}_{\mathrm{s}} \rightarrow \Phi \Phi:$

Measure hadronic penguin $\mathrm{B}_{\mathrm{s}} \rightarrow \Phi \Phi$.

(NP $\rightarrow$ contribution to decay mode?)
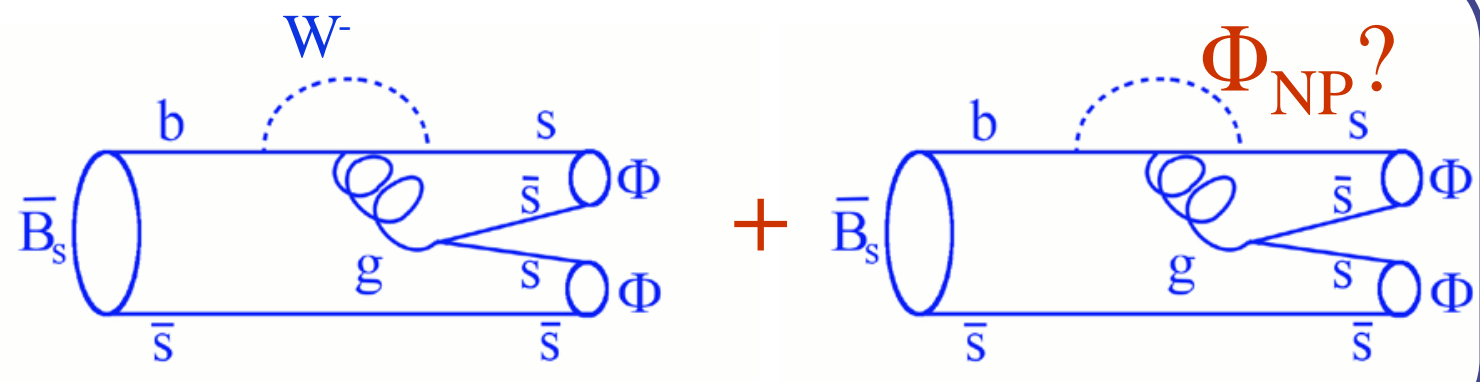


\section{CP-violation in the $B_{s}$-system}

Decay into a final state $f$ with $\mathrm{CP} \mathrm{f} \rightarrow \eta_{\mathrm{f}} \mathrm{f}$

(assume only one amplitude contributes to decay)

CP-asymmetry:

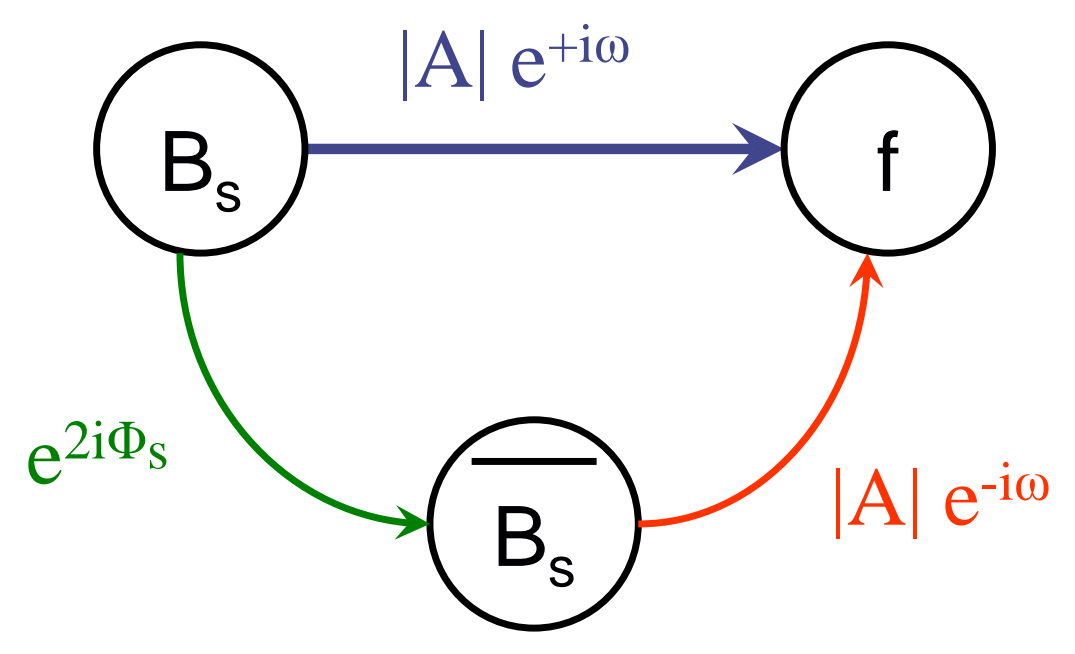

$\begin{aligned} A_{C P}(t) & =\frac{\Gamma\left(\overline{B_{s}^{0}}(t) \rightarrow f_{C P}\right)-\Gamma\left(B_{s}^{0}(t) \rightarrow f_{C P}\right)}{\Gamma\left(\overline{B_{s}^{0}}(t) \rightarrow f_{C P}\right)+\Gamma\left(B_{s}^{0}(t) \rightarrow f_{C P}\right)} \\ & =-\frac{\eta_{f} \sin \left(\phi_{s}-2 \omega\right) \sin \left(\Delta m_{s} t\right)}{\cosh \left(\frac{\Delta \Gamma_{s} t}{2}\right)-\eta_{f} \cos \phi_{s} \sinh \left(\frac{\Delta \Gamma_{s} t}{2}\right)}\end{aligned}$

For $\mathrm{B}_{\mathrm{s}} \rightarrow \mathrm{J} / \psi \phi:$ $\omega \approx 0$ 


\section{$\Phi_{\mathrm{s}}$ and $\Delta \Gamma_{\mathrm{s}}^{\prime}$}

9

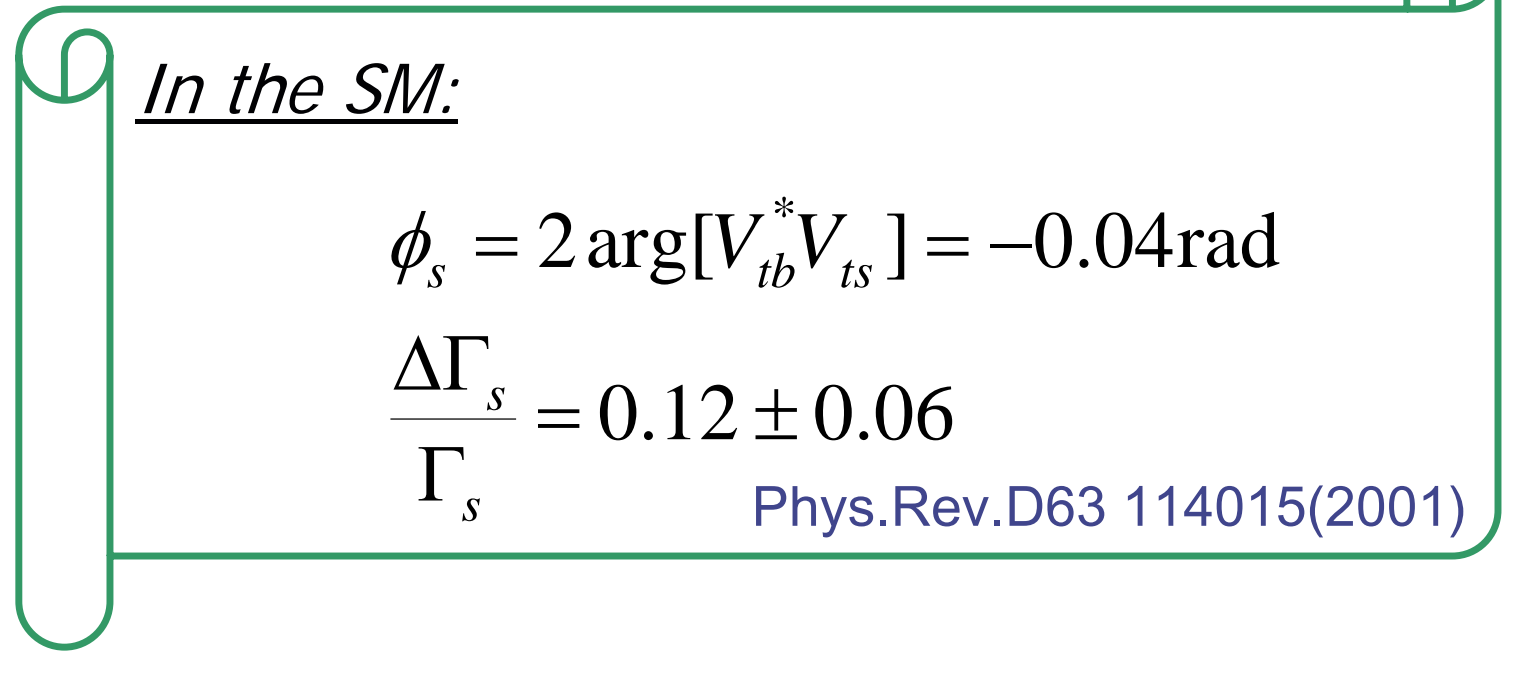

9

If new physics contributes to $\underline{B}_{s}$ mixing:

$$
\begin{aligned}
& \phi_{s} \rightarrow \phi_{s}+\phi_{N P} \\
& \Delta \Gamma_{s} \rightarrow \Delta \Gamma_{s} \cos \left(\phi_{s}\right)
\end{aligned}
$$

Any sizeable $\mathrm{CP}$ violation in $\mathrm{B}_{\mathrm{s}} \rightarrow \mathrm{J} / \psi \Phi$ or $\mathrm{B}_{\mathrm{s}} \rightarrow \Phi \Phi$ is a clear sign for NP! 


\section{$\mathrm{B}_{\mathrm{s}} \rightarrow J / \psi\left(\mu^{+} \mu^{-}\right) \phi\left(\mathrm{K}^{+} \mathrm{K}^{-}\right)$}

Advantages: High branching ratio

Good experimental signature

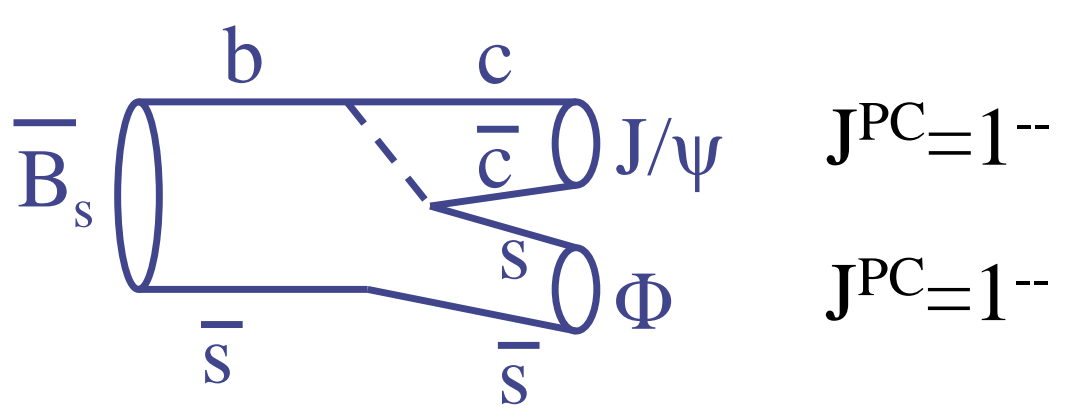

$\mathrm{CP}(\mathrm{J} / \psi \phi)=\mathrm{CP}(\mathrm{J} / \psi) \operatorname{CP}(\phi)(-1)^{\mathrm{L}}$

$\left.\Rightarrow \begin{array}{l}\mathrm{L}=0,2 \text { : CP even } \\ \mathrm{L}=1 \text { : CP odd }\end{array}\right\} \begin{aligned} & \text { Final state is } \\ & \text { a mixture of } \\ & \text { CP even/odd }\end{aligned}$

$\Rightarrow$ Angular analysis needed to identify CP even and CP odd states! 


\section{$\mathrm{B}_{\mathrm{s}} \rightarrow J / \psi\left(\mu^{+} \mu^{-}\right) \phi\left(\mathrm{K}^{+} \mathbb{K}^{-}\right)$}

Use angle $\theta_{\text {tr }}$ between $\mu^{+}$and normal of $\phi$ decay plane:

$$
\begin{gathered}
\frac{d \Gamma}{d \cos \Theta_{t r}} \propto\left(A_{0}(t)^{2}+\left|A_{2}(t)\right|^{2}\right)^{3} \frac{3}{8}\left(1+\cos ^{2} \Theta_{t r}\right) \\
+\mid A_{1}(t)^{2} \frac{3}{4} \sin ^{2} \Theta_{t r} \\
\left|A_{0,2}(t)^{2}=\right| A_{0,2}(0)^{2}\left(e^{\Gamma_{L} t}-e^{\bar{\Gamma} t} \sin \left(\Phi_{s}\right) \sin \left(\Delta m_{s} t\right)\right) \\
\left|A_{1}(t)\right|^{2}=\left|A_{1}(0)\right|^{2}\left(e^{\Gamma_{L} t}+e^{\bar{\Gamma} t} \sin \left(\Phi_{s}\right) \sin \left(\Delta m_{s} t\right)\right)
\end{gathered}
$$

\section{CP-odd fraction:}

$R_{T} \equiv \frac{\left|A_{\perp}(t)\right|^{2}}{\sum_{f=0, \|, \perp}\left|A_{f}(t)\right|^{2}}$

$$
R_{T}(t=0) \sim 20 \% \text { (CDF, D0) }
$$$$
R_{T}=0 \rightarrow \text { CP even }
$$

$R_{T}=0.5 \rightarrow$ Maximum dilution
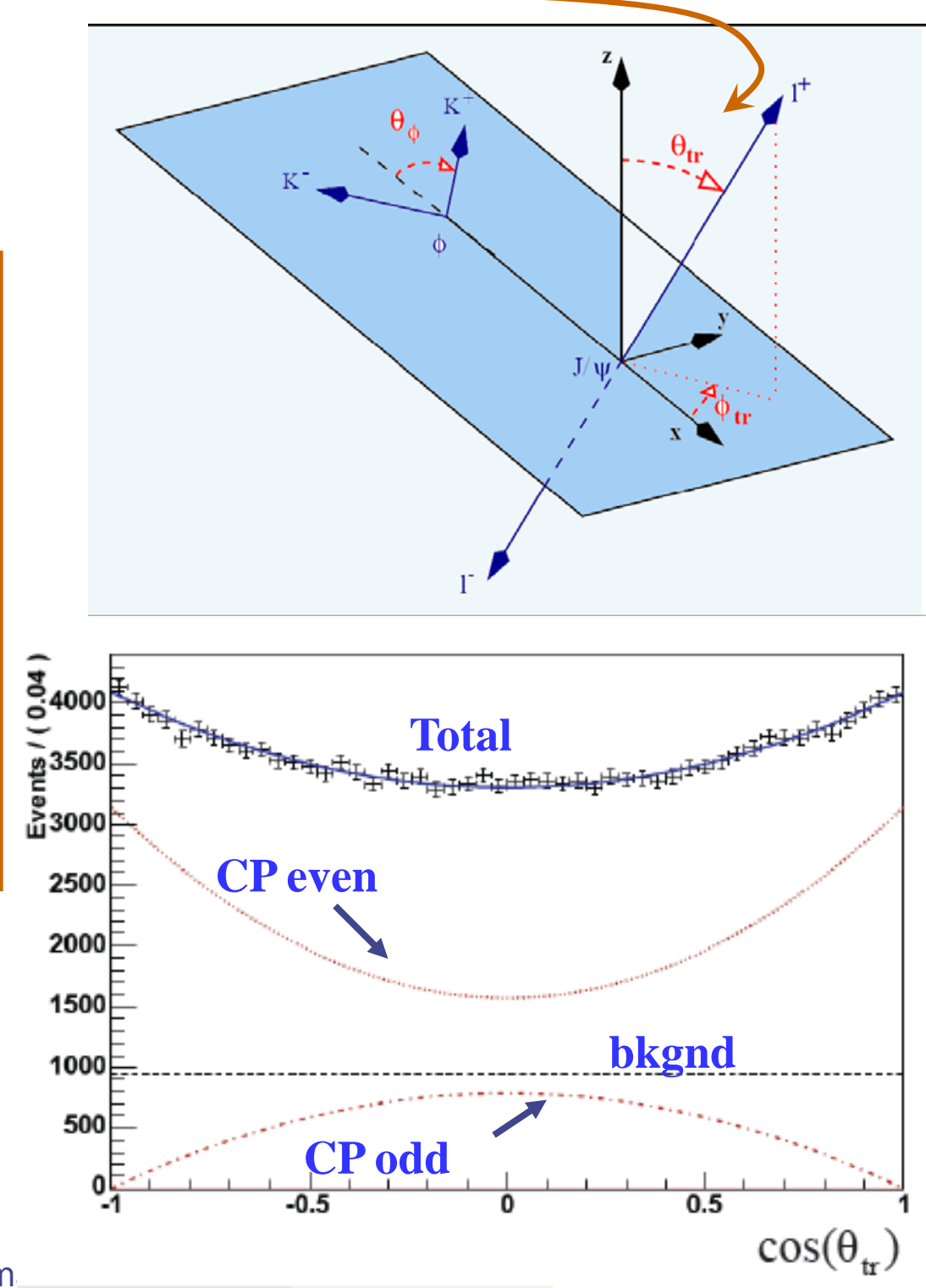


\section{Selection and signal decay rate}

\begin{tabular}{|ll|}
\hline$\cdot$ Yield: & $130 \mathrm{k}$ events per $2 \mathrm{fb}^{-1}$ \\
$\cdot \mathrm{B} / \mathrm{S}:$ & 0.12 \\
$-<\delta_{\mathrm{t}}>:$ & $36 \mathrm{fs}$ \\
$-\sigma_{\text {Mass }}:$ & $14 \mathrm{Mev} / \mathrm{c}^{2}$ \\
$-\mathrm{W}_{\text {tag: }}$ & $33 \%$ \\
$\cdot \boldsymbol{\varepsilon}_{\text {tag }}:$ & $57 \%$ \\
\hline
\end{tabular}

\section{Signal decay rates including:}

- Trigger and selection bias on $\tau$

- Background parametrization

- Mass resolution

- Proper time resolution

- Tagging efficiency and dilution

- Transversity angle distribution

$$
\mathrm{B}_{\mathrm{s}} \rightarrow \mathrm{J} / \psi \Phi:
$$

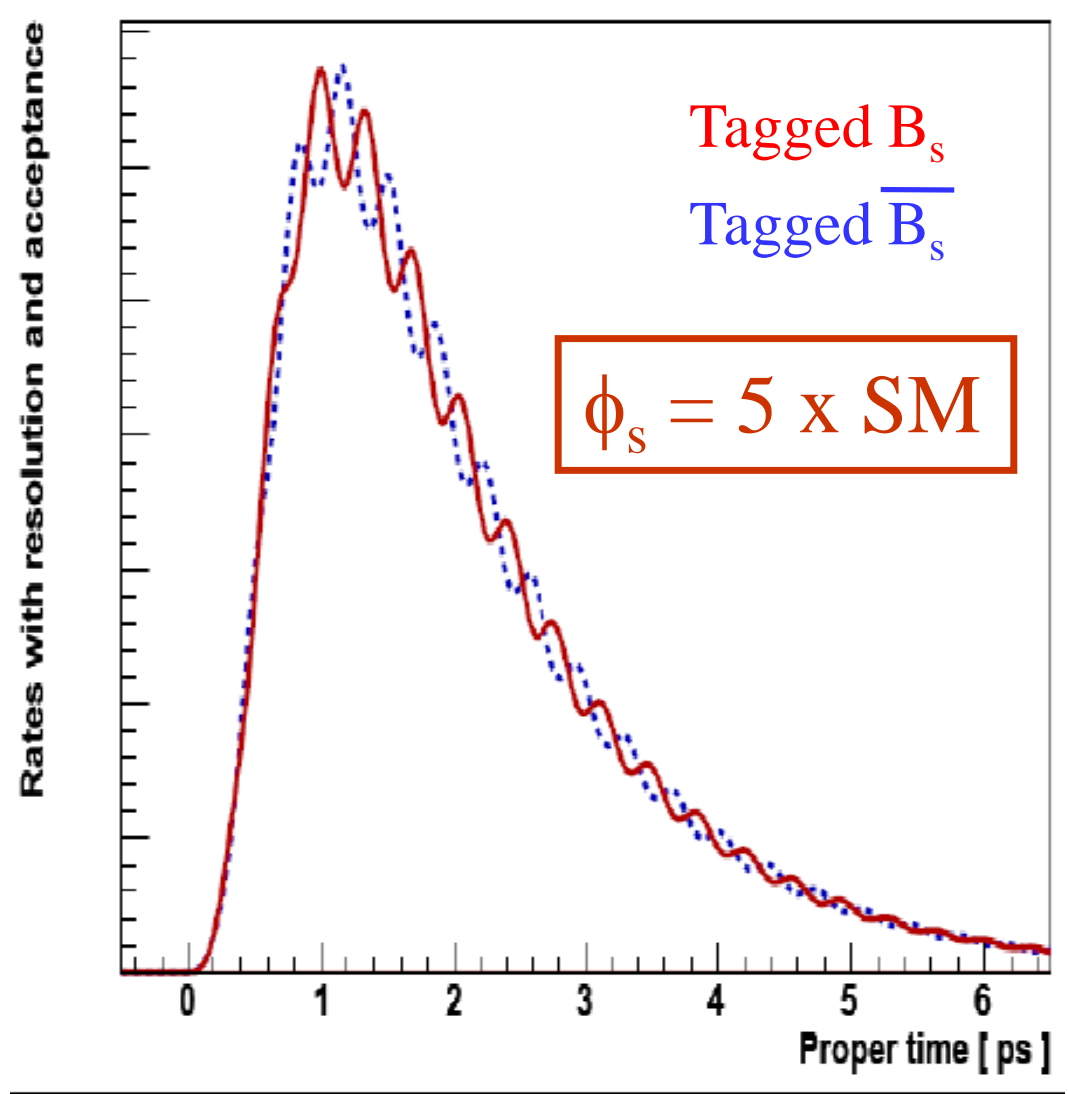




\section{Projection for $\Phi_{s}$ and $\Delta \Gamma_{s}$ with $2 \mathrm{fb}^{-1}$}

$\left.\begin{array}{|c|c|c|}\hline \text { Parameter } & \text { Exp. error } & \text { Channel } \\ \phi_{\mathrm{s}} & 0.023 \mathrm{rad} & \mathrm{B}_{\mathrm{s}} \rightarrow \mathrm{J} / \psi\left(\mu^{+} \mu^{-}\right) \Phi\left(\mathrm{K}^{+} \mathrm{K}^{-}\right) \\ \Delta \Gamma / \Gamma & 0.0092 & \mathrm{~B}_{\mathrm{s}} \rightarrow \mathrm{J} / \psi\left(\mu^{+} \mu^{-}\right) \Phi\left(\mathrm{K}^{+} \mathrm{K}^{-}\right) \\ \hline \Delta \mathrm{m}_{\mathrm{s}} & 0.007 \mathrm{ps}^{-1} & \mathrm{~B}_{\mathrm{s}} \rightarrow \mathrm{D}_{\mathrm{s}}^{-}\left(\mathrm{K}^{+} \mathrm{K}^{-} \pi^{-}\right) \pi^{+} \\ \hline \mathrm{w}_{\text {tag }} & 0.0036 & \mathrm{~B}_{\mathrm{s}} \rightarrow \mathrm{D}_{\mathrm{s}}^{-}\left(\mathrm{K}^{+} \mathrm{K}^{-} \pi^{-}\right) \pi^{+}\end{array}\right\} \begin{aligned} & \text { Control } \\ & \text { channel } \\ & \text { only }\end{aligned}$

Sensitivity can be improved by adding more channels. Using $\mathrm{B}_{\mathrm{s}} \rightarrow \mathrm{J} / \psi \eta, \mathrm{B}_{\mathrm{s}} \rightarrow \eta_{\mathrm{C}} \phi, \mathrm{B}_{\mathrm{s}} \rightarrow \mathrm{D}_{\mathrm{s}} \mathrm{D}_{\mathrm{s}}$ gives $\sigma_{\Phi}=0.021 \mathrm{rad}$.

CP-Eigenstates 


\section{$\mathrm{B}_{\mathrm{s}} \rightarrow \phi \phi:$ Sensitvity to $\phi_{\mathrm{s}}$}

LHCb profits from excellent PID and hadronic trigger!

DExpected yield: 4000 events per $2 \mathrm{fb}^{-1}$

$>\mathrm{BG}$ estimate limited by MC statistics: $0.4<\mathrm{B} / \mathrm{S}<2.1$ at $90 \% \mathrm{CL}$

$>$ Sensitivity to $\phi_{\mathrm{s}}$ is $0.1 \mathrm{rad}$ at $2 \mathrm{fb}^{-1}$.

$>$ No significant variation as a function of input $\phi_{\mathrm{s}}, \mathrm{R}_{\mathrm{t}}$ and proper time resolution.
Distribution from $500 \mathrm{MC}$ experiments:

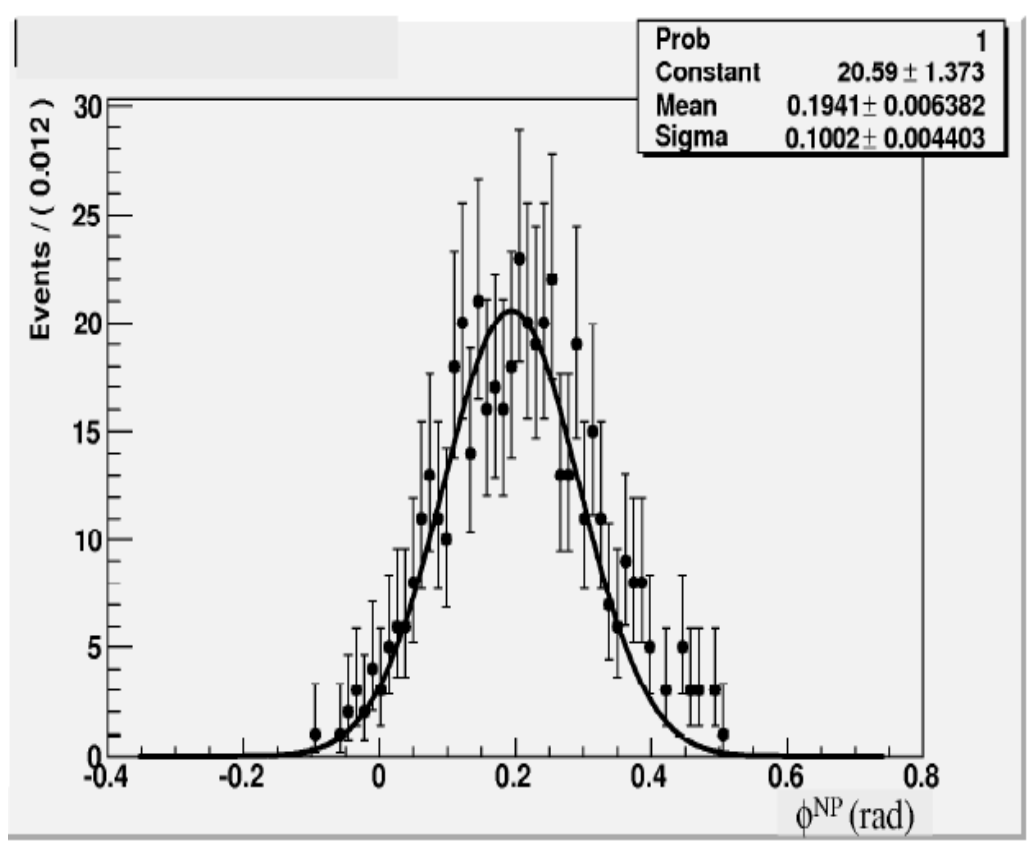

Baseline physics inputs (varied for robustiness) to sensitivity studies

$$
\begin{array}{cc}
\phi^{N P}=0.2 & R_{t}=\left|A_{\perp}\right|^{2} /\left(\left|A_{\perp}\right|^{2}+\left|A_{0}\right|^{2}+\left|A_{/ /}\right|^{2}\right)=0.25 \\
\delta_{/ /}=0, \delta_{0}=\pi & \left.R_{p}=\left|A_{/ /}\right|^{2} /\left|A_{\perp}\right|^{2}+\left|A_{0}\right|^{2}+\left|A_{/ /}\right|^{2}\right)=0.25 \\
\Gamma_{\mathrm{s}}=0.67 \mathrm{ps}^{-1}, \Delta m_{s}=17 \mathrm{ps}^{-1} & R_{p, t} \text { values motivated by polarisation } \\
\Delta \Gamma_{\mathrm{s}} / \Gamma_{\mathrm{s}}=15 \% & \text { measurements of } B^{0} \rightarrow \phi K^{* 0}
\end{array}
$$




\section{NP by Tree $\leftrightarrow$ Penguin comparison}

$\underline{B}_{d}$-system:
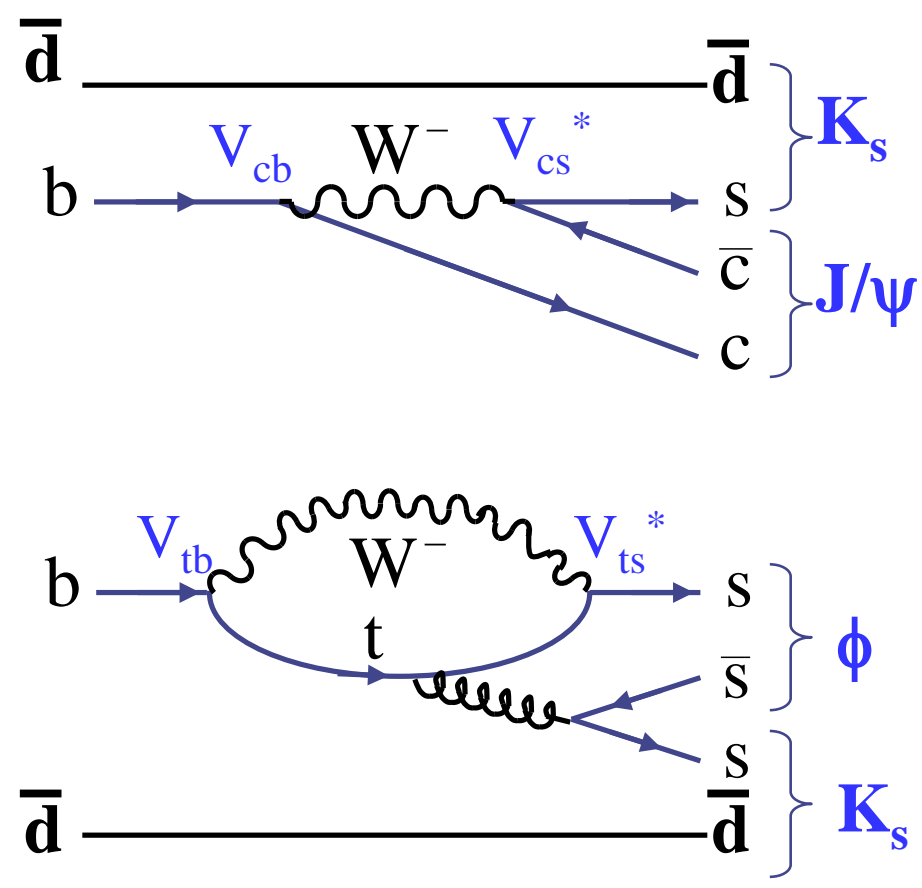

$\Phi_{\mathrm{d}}($ tree $)-\Phi_{\mathrm{d}}($ penguin $)=\delta \boldsymbol{\Phi}_{\mathrm{d}}(\mathbf{N P})$

\section{B-factories:}

Currently: $\delta \beta=8^{\circ}(2.6 \sigma)$
Bs -system:

Tree

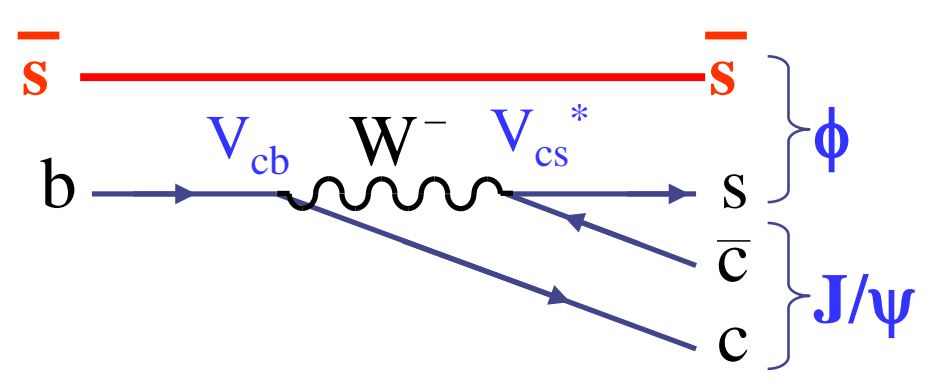

Penguin

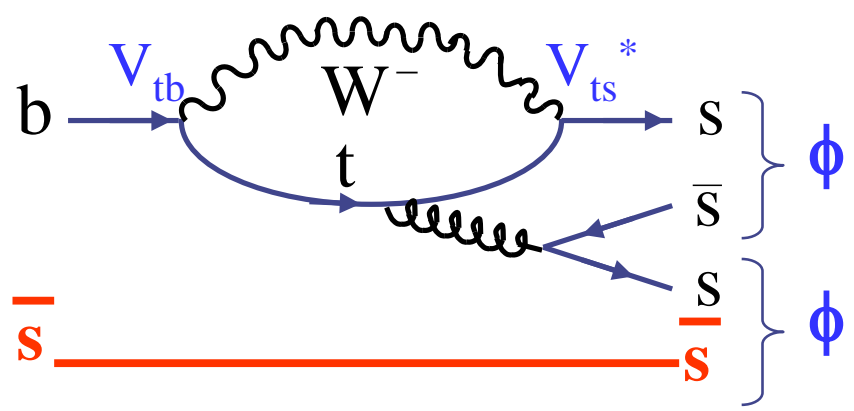

$\Phi_{\mathrm{s}}($ tree $)-\Phi_{\mathrm{s}}($ penguin $)=\delta \Phi_{\mathrm{s}}(\mathbf{N P})$ And:

$\Phi_{\mathrm{s}}(\mathrm{SM})$ small!

LHCb-projection $\left(2 \mathrm{fb}^{-1}\right): \sigma\left(\phi_{\mathrm{s}}\right)=6$ 


\section{Rare decays}

\begin{tabular}{|c|c|c|}
\hline Decay & Sensitivity to & Example for model \\
\hline 1. $\mathrm{B}_{\mathrm{d}, \mathrm{s}} \rightarrow \mu^{+} \mu^{-}$ & $a^{- \text {large } \tan \beta}$ & CMSSM \\
\hline 2. $B_{d} \rightarrow K^{* 0} \mu \mu$ & $\begin{array}{l}\text { - small } \tan \beta \\
\text { - right handed currents }\end{array}$ & $\begin{array}{l}\text { non-MFV MSSM } \\
\text { MIA MSSM } \\
\text { SUGRA }\end{array}$ \\
\hline $\begin{array}{l}\text { 3. } \mathbf{B}_{\mathbf{u}} \rightarrow \mathbf{K}^{+} \mathbf{l l} \\
\quad \text { in combination } \\
\quad \text { with 1.) }\end{array}$ & $\begin{array}{l}\text { - (pseudo)-scalar } \\
\text { couplings }\end{array}$ & MFV \\
\hline
\end{tabular}




\section{1. $B_{d, s} \rightarrow \mu^{+} \mu^{-}$}

\section{SM expectation:}

$\mathrm{BR}\left(\mathrm{B}_{\mathrm{s}} \rightarrow \mu^{+} \mu^{-}\right)=\left(\begin{array}{ll}3.4 & 0.4\end{array}\right) \times 10^{-9}$

$\operatorname{BR}\left(B_{d} \rightarrow \mu^{+} \mu^{-}\right)=(1.0 \pm 0.5) \times 10^{-10}$

World best limit by D0:

$\mathrm{BR}\left(\mathrm{B}_{\mathrm{s}} \rightarrow \mu^{+} \mu^{-}\right)<7.5 \times 10^{-8} @ 90 \% \mathrm{CL}$

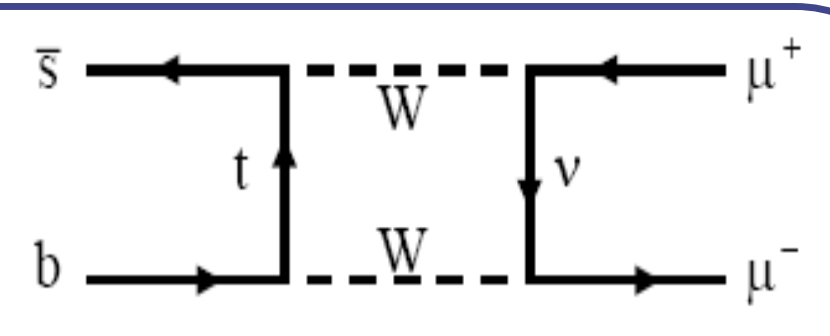

\section{In Supersymmetry:}

Large contributions e.g. by

Higgs penguins $\sim \tan ^{6} \beta$, i.e.

$\operatorname{BR}\left(B_{d, s} \rightarrow \mu+\mu-\right)$ is very

sensitive to high values

of $\tan \beta$.
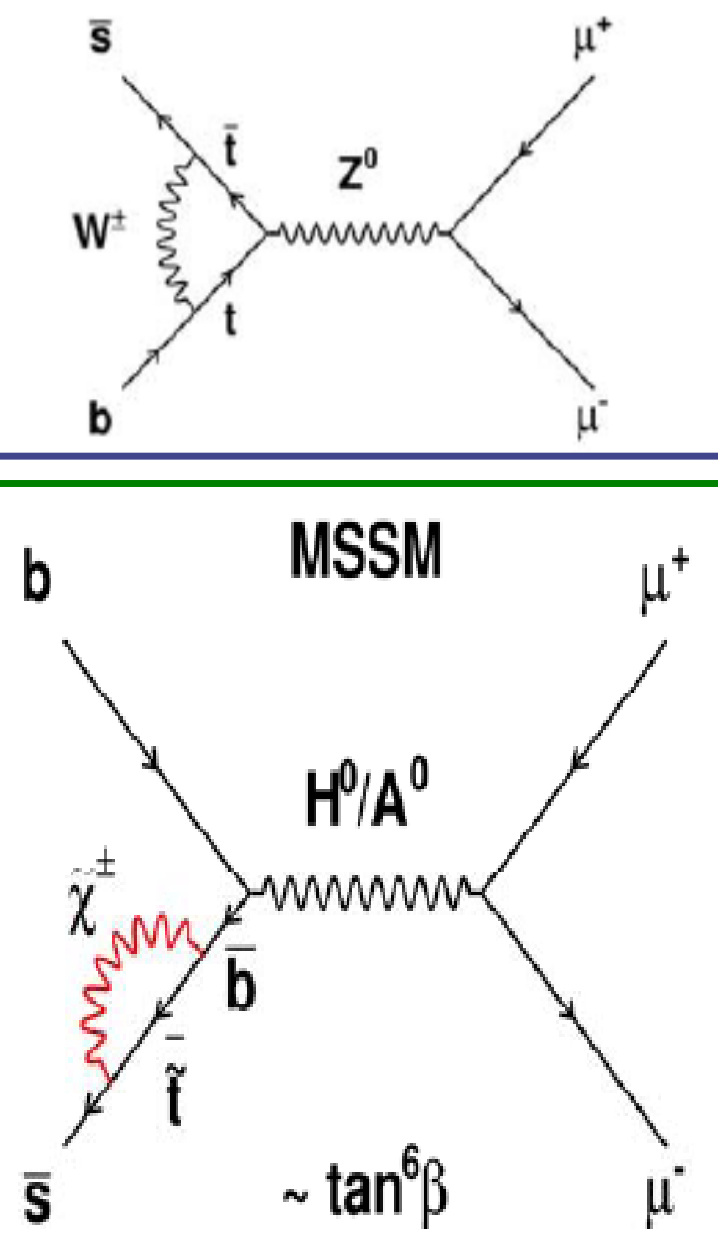


\section{Sensitivity by LHCb}

Main background:

$>$ Combinatoric $\mathrm{b} \rightarrow \mu, \mathrm{b} \rightarrow \mu$

$>\mathrm{B}_{\mathrm{c}} \rightarrow \mathrm{J} / \psi\left(\mu^{+} \mu^{-}\right) \mu v$

$>\mathrm{B}_{\mathrm{d}, \mathrm{s}} \rightarrow \mathrm{h}^{+} \mathrm{h}^{-}$
Adressed by excellent mass resolution (18MeV), vertex resolution and particle ID

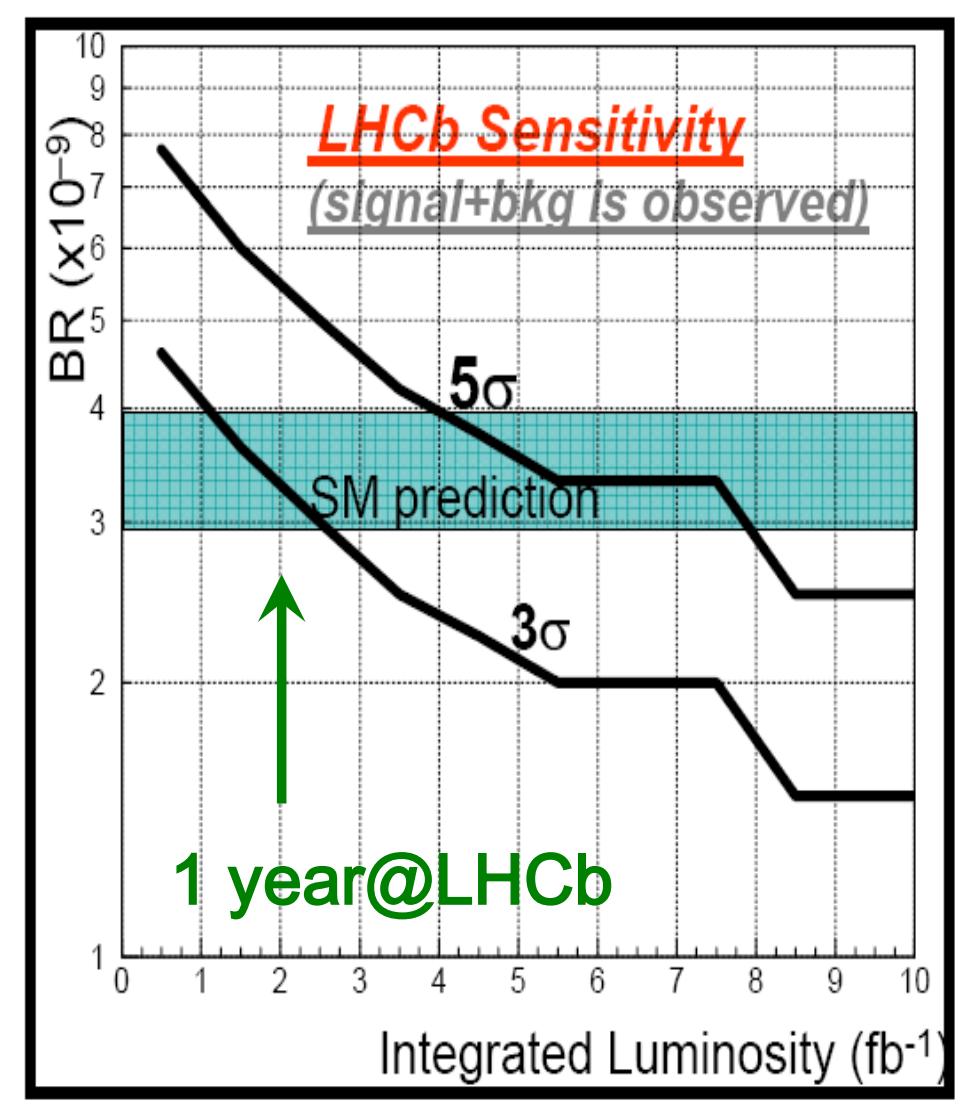

$\operatorname{BR}\left(B_{s} \rightarrow \mu^{+} \mu^{-}\right)$in CMSSM as a

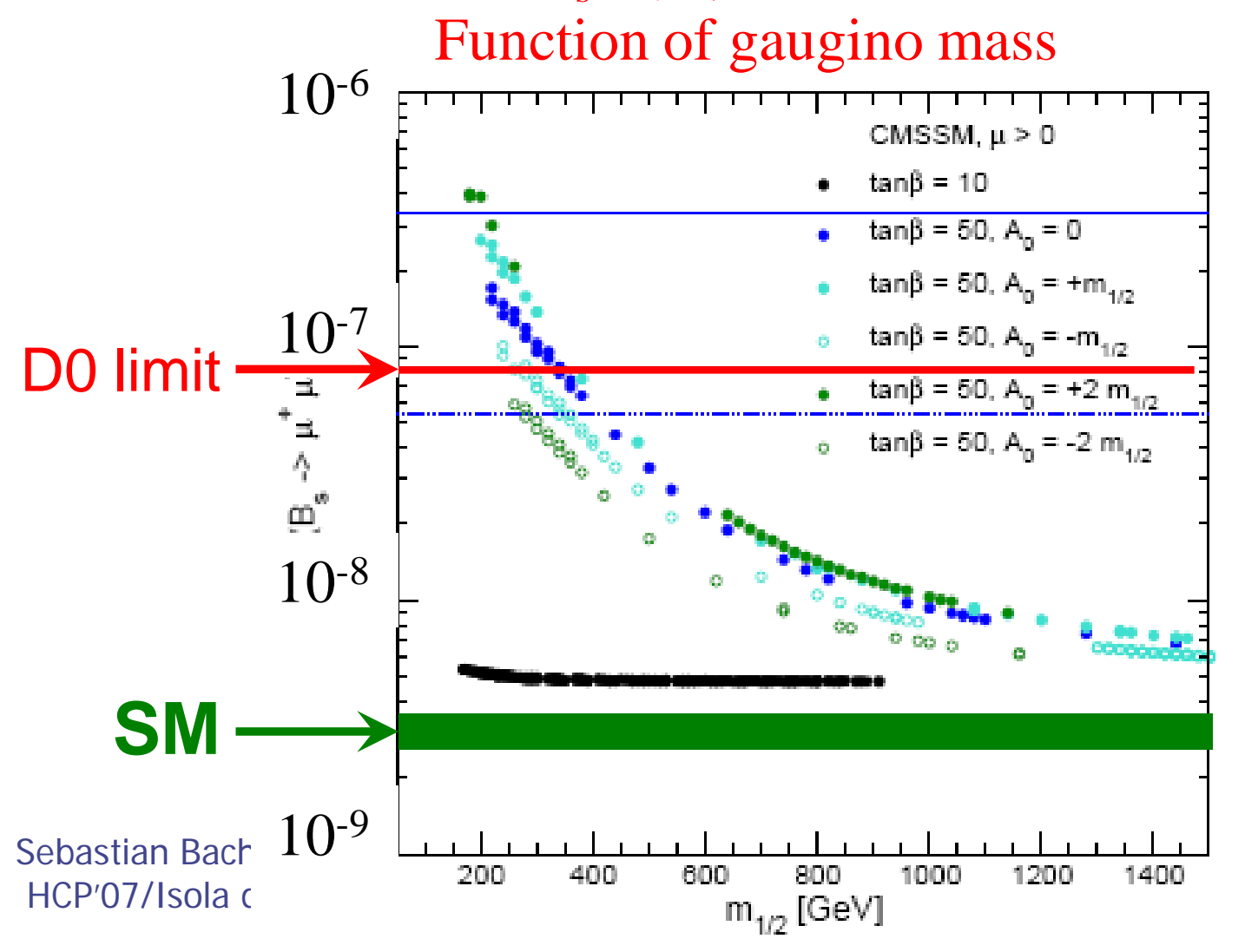




\section{3.) $\mathrm{B}_{\mathrm{u}}^{+} \rightarrow \mathbb{K}^{+} \mathrm{II}$}

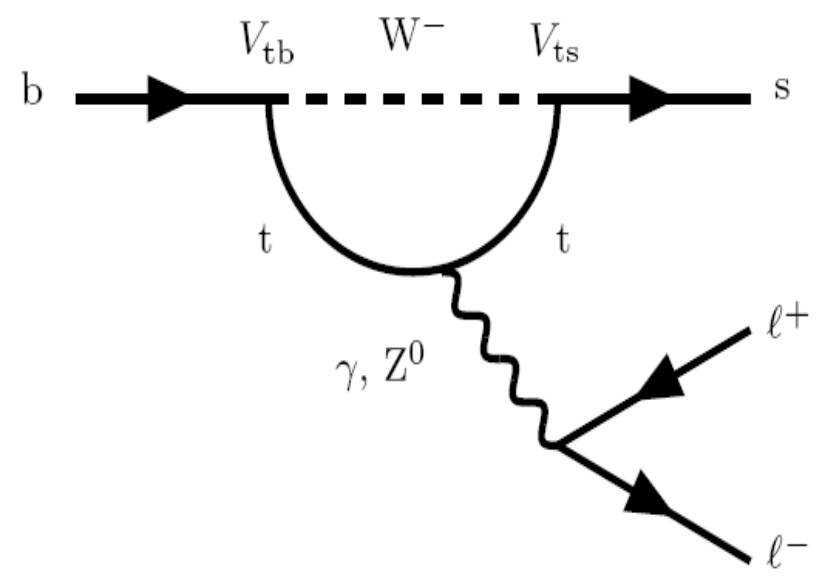

Use the ratio

$$
R_{\mathbf{X}}=\frac{\int_{4 m_{\mu}^{2}}^{q_{\text {max }}^{2}} s \frac{d \Gamma\left(\mathbf{B} \rightarrow \mathbf{X} \mu^{+} \mu^{-}\right)}{\mathrm{d} s}}{\int_{4 m_{\mu}^{2}}^{q_{\text {max }}^{2}} \mathrm{~d} s \frac{d \Gamma\left(\mathbf{B} \rightarrow \mathbf{X} \mathbf{X e}^{+} \mathbf{e}^{-}\right)}{\mathrm{d} s}} \stackrel{M M}{=}\left\{\begin{array}{lll}
1.000 \pm 0.001 & \mathbf{X}=\mathbf{K} & \sim \mathrm{m}_{1} \\
0.991 \pm 0.002 & \mathbf{X}=\mathbf{K}^{*}
\end{array}\right.
$$

Hiller \& Krüger, PRD69 (2004) 074020) 


\section{$\mathrm{B}_{\mathrm{u}}^{+} \rightarrow \mathrm{K}^{+}$II with $2 \mathrm{fb}^{-1}$}
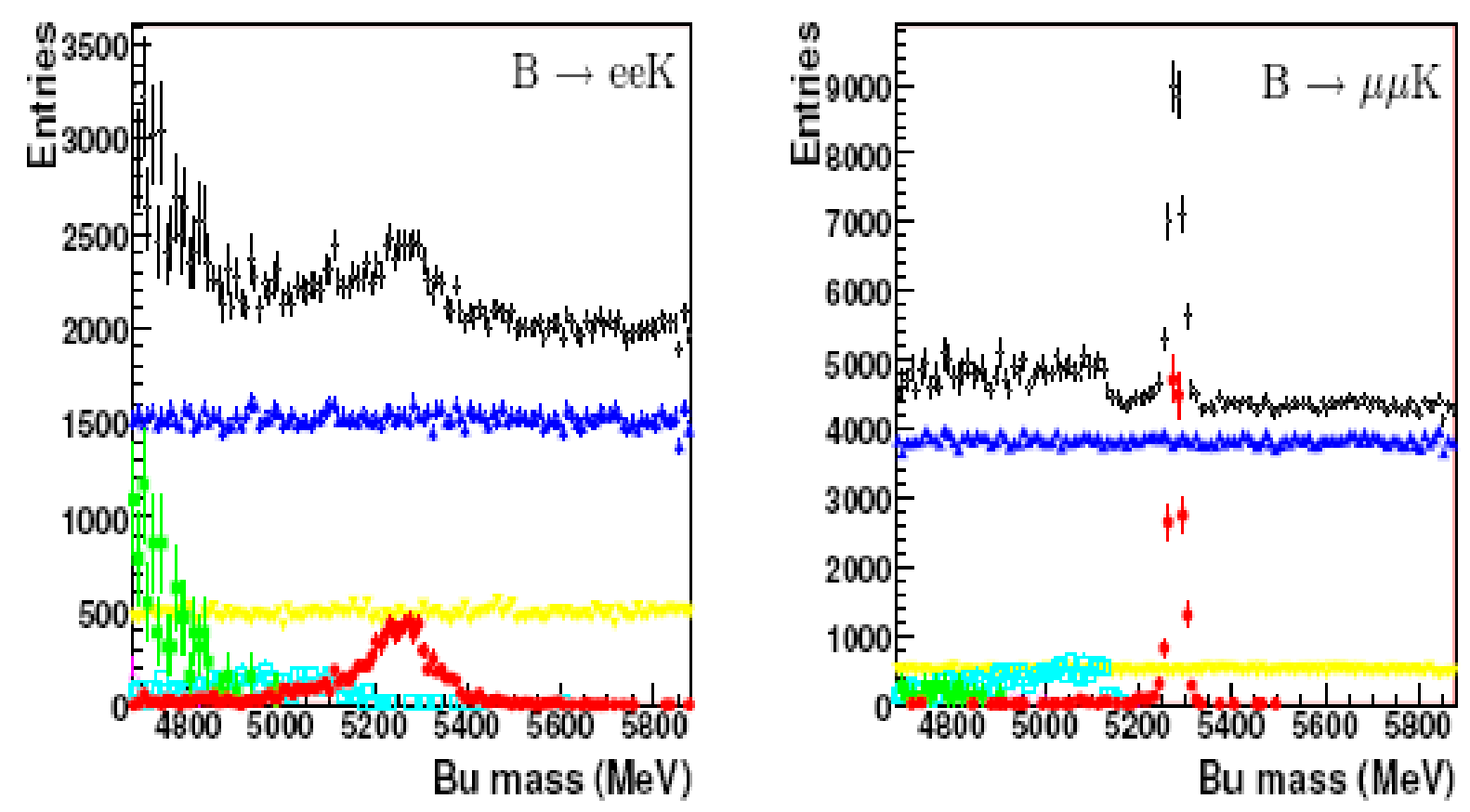

$>$ Takes into account an inclusive di-lepton trigger

$>$ applies Bremsstrahlung corrections

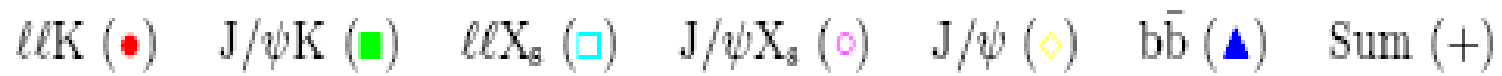

\begin{tabular}{l|lll} 
& Signal & Mean & Sigma \\
\hline eeK & 34934 & $5245 \mathrm{MeV}$ & $74 \mathrm{MeV}$ \\
$\mu \mu \mathrm{K}$ & $1550 \pm 50$ & $5279 \mathrm{MeV}$ & $15 \mathrm{MeV}$
\end{tabular}

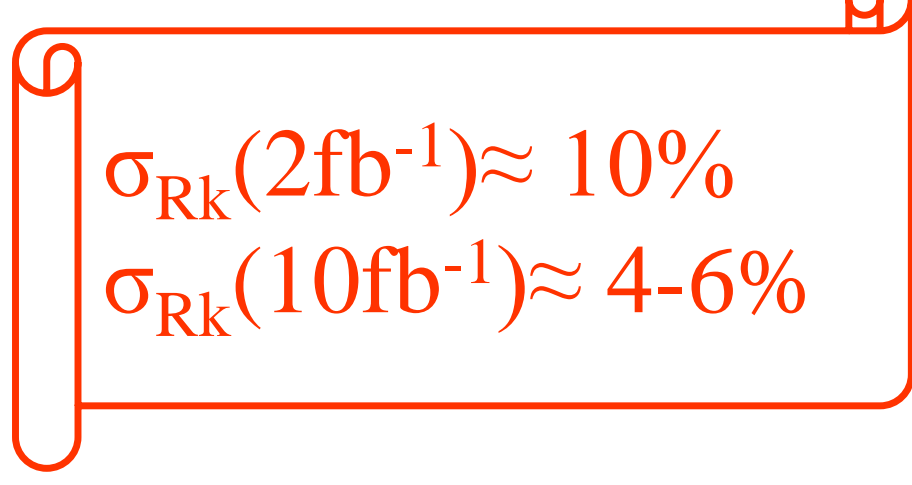




\section{$\mathrm{R}_{\mathrm{k}}$ in a MFV model}

\section{Assume}

o Right handed currents are negligible.

o (Pseudo-)scalar couplings lepton masses

o No CP-phases beyond the SM

$$
\mathrm{R}_{\mathrm{k}}-1 \sim \mathrm{BR}\left(\mathrm{B}_{\mathrm{s}} \rightarrow \mu \mu\right)
$$

Hiller \& Krüger, PRD69 (2004) 074020)

LHCb projection if SM is holds

But we hope for

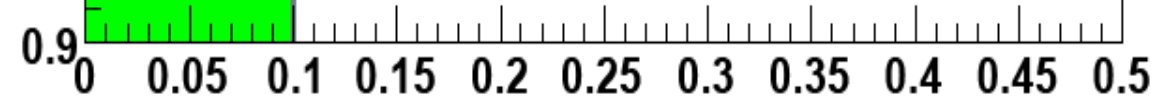
something else...

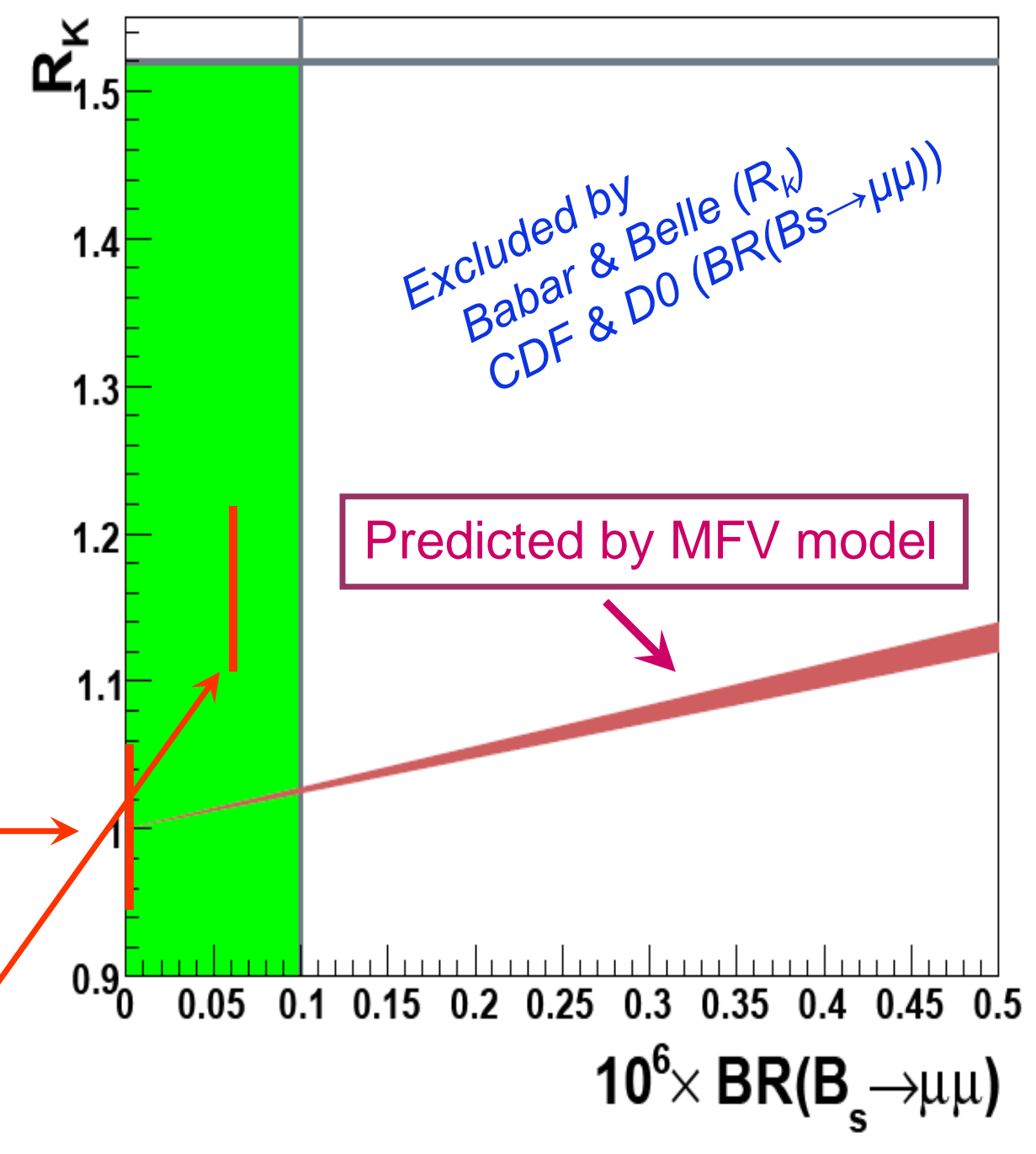




\section{Conclusion}

$>$ LHCb is on a good track to take first data soon.

$>$ It has a wide potential to search for New Physics complementary to new particle searches.

$>$ Searches allow to

- find New Physics in model independent analysis,

e.g. by measuring $\mathrm{B}_{\mathrm{s}}$-mixing and related $\mathrm{CP}$-asymmetries.

- pin-down the nature of New Physics e.g. by the study of rare decays.

$>$ The challenge is to achieve that performance with real data! 


\section{Backup slides}




\section{Performance: Vertex locator}
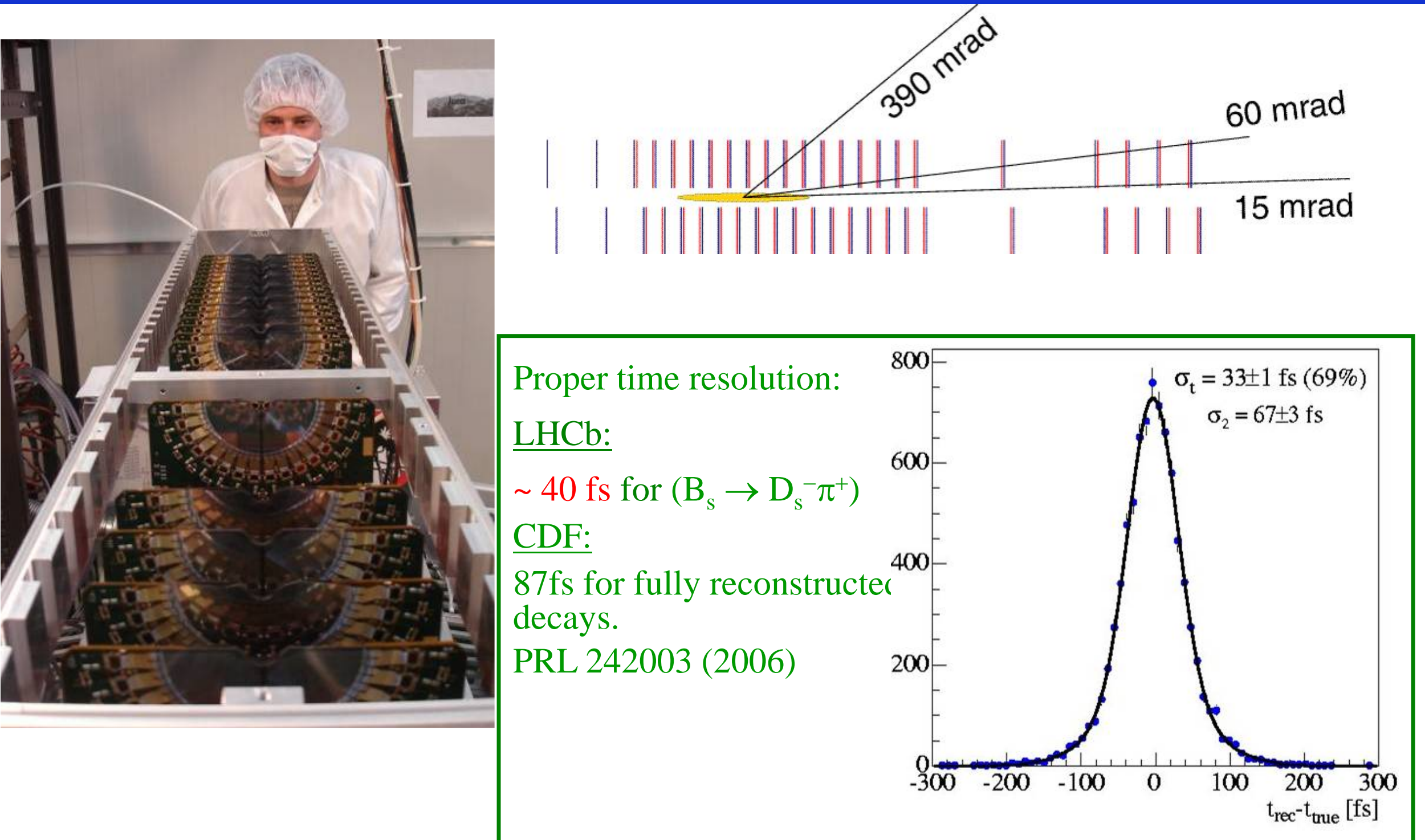


\section{Tracking}

Momentum resolution
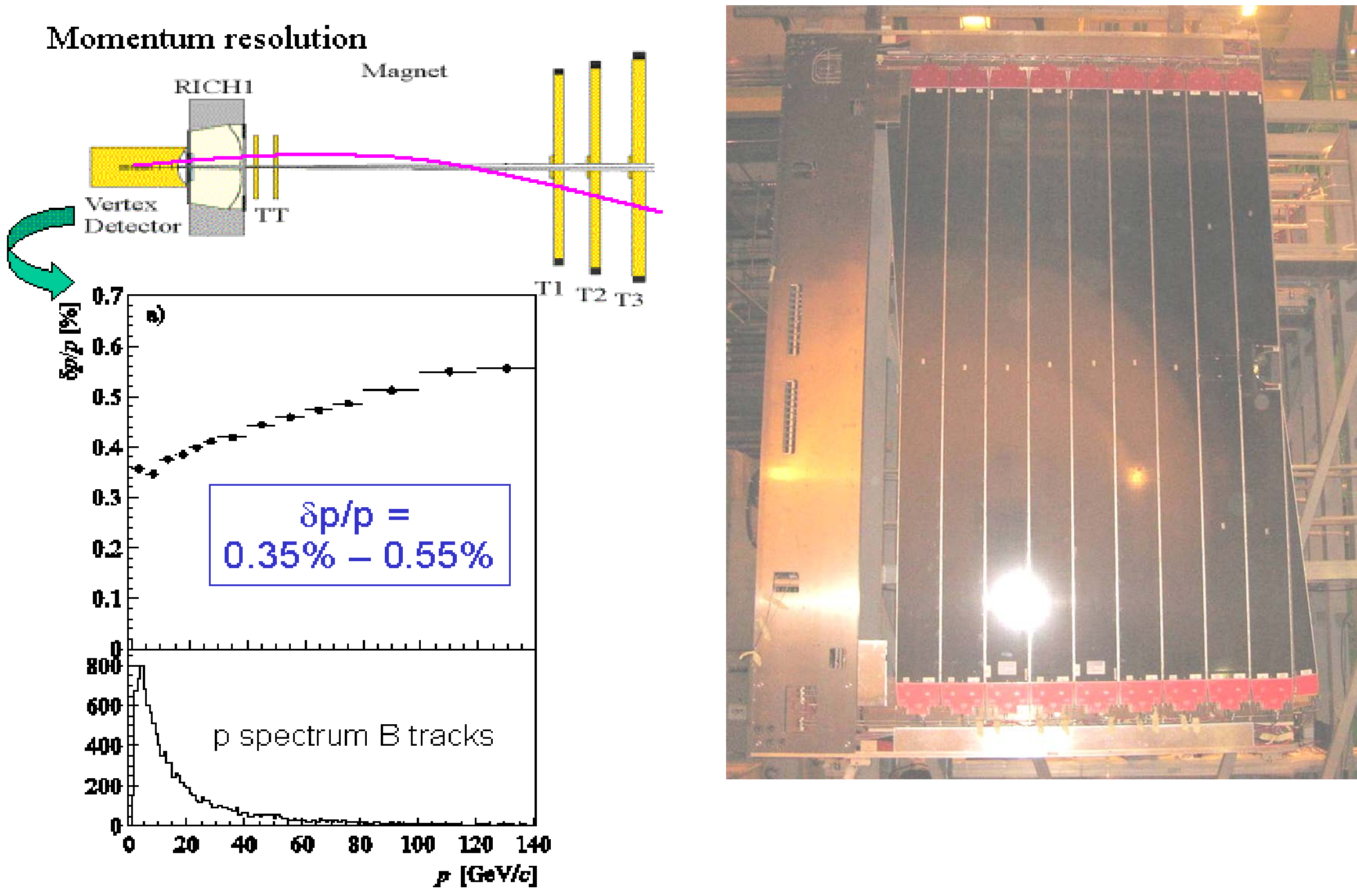


\section{Performance: Particle Identification}

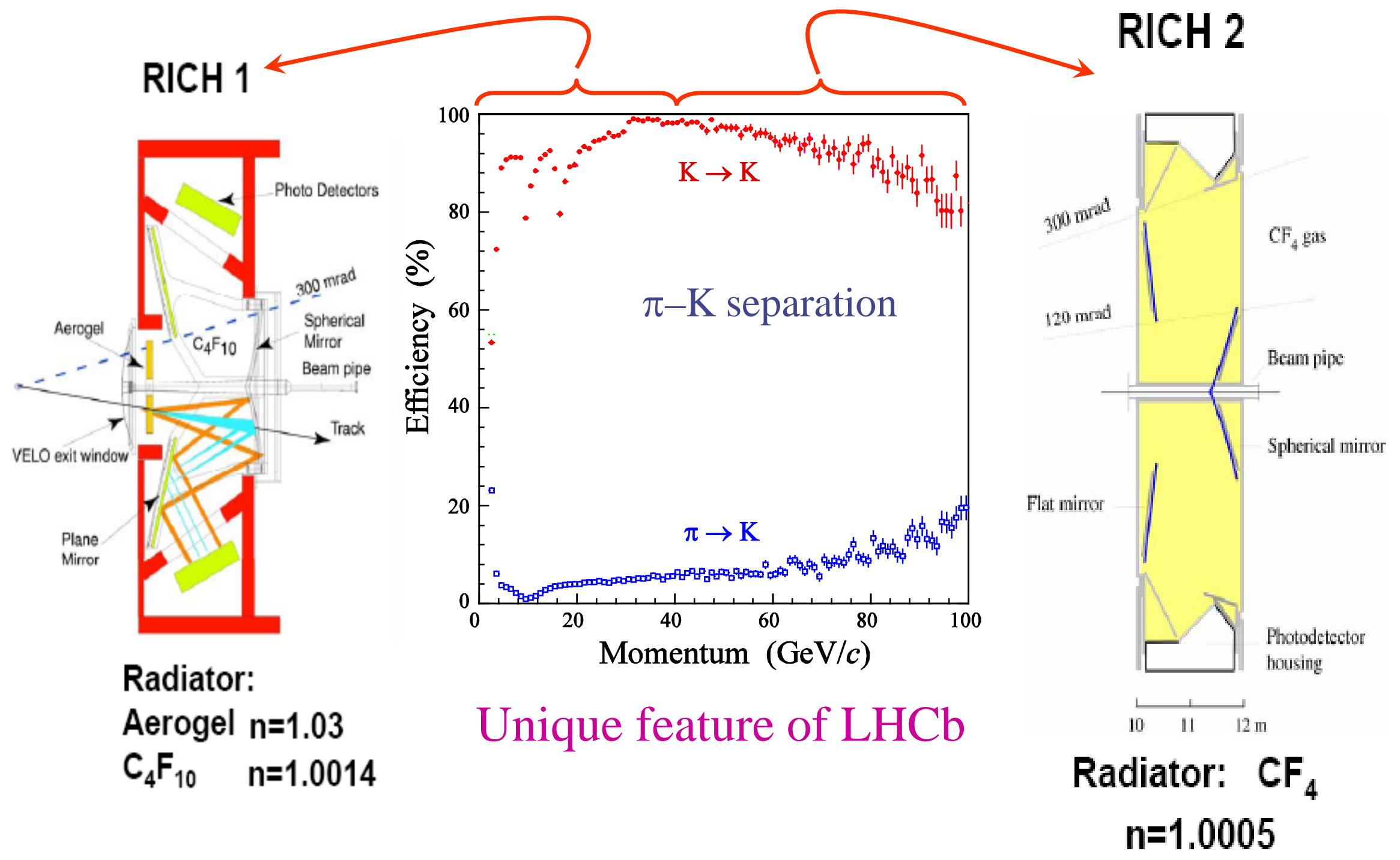




\section{Performance of VeLo}

\section{Impact parameter resolution:}

$$
\delta \mathrm{IP}=14 \mu \mathrm{m}+35 \mu \mathrm{m} / \mathrm{p}_{\mathrm{t}}
$$

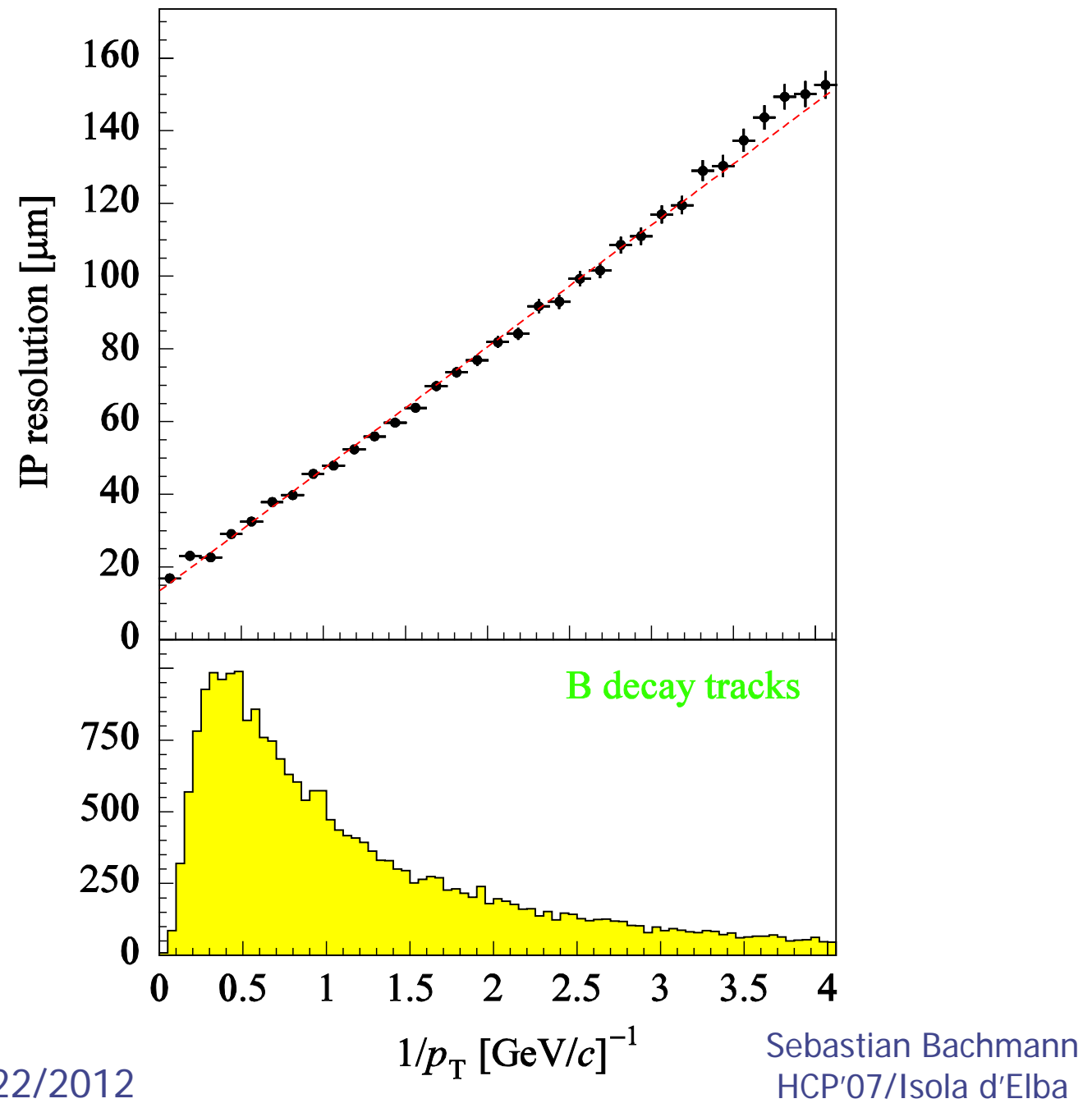

Proper time resolution:

$\sim 40$ fs $\left(\mathrm{B}_{\mathrm{s}} \rightarrow \mathrm{D}_{\mathrm{s}}^{-} \pi^{+}\right)$

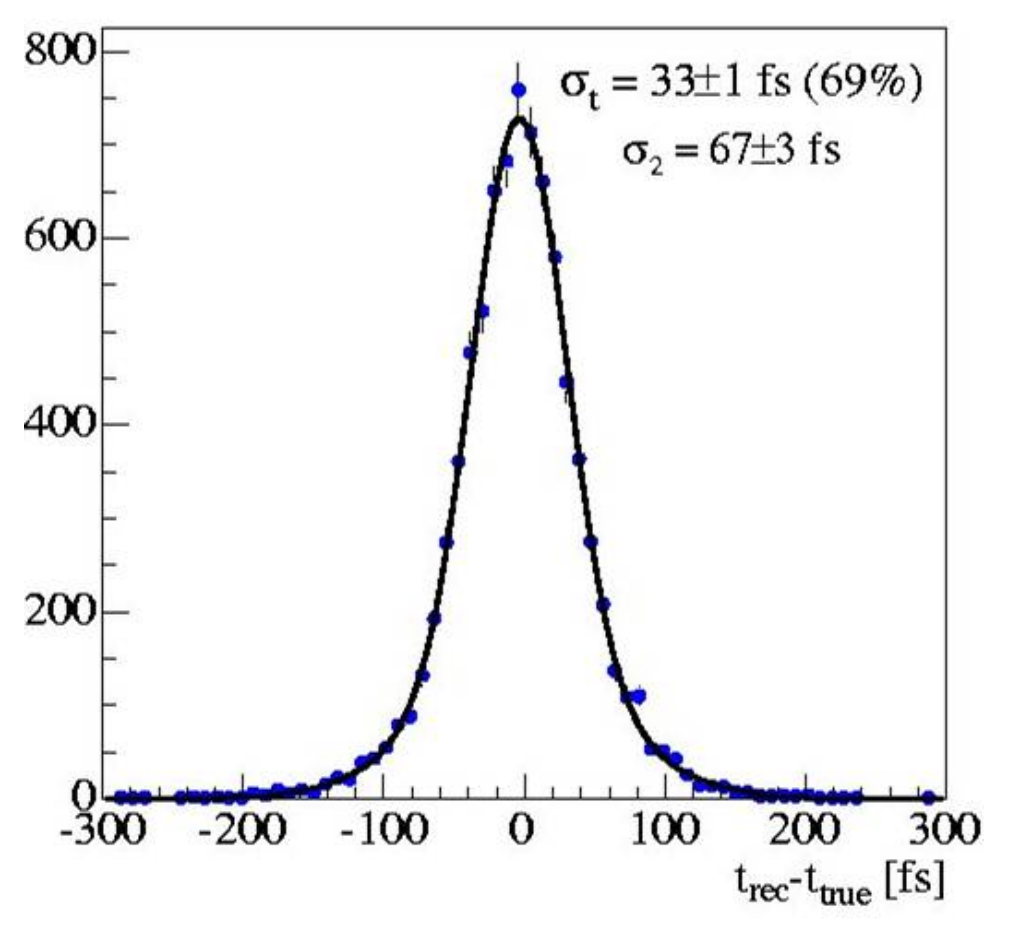




\section{Calorimeter}

\section{Calorimeter system to}

identify electrons, hadrons and neutrals

Important for the first level of the trigger

o Scintillating Pad Detector / PreShower

o Electromagnetic calorimeter o Hadron Calorimeter

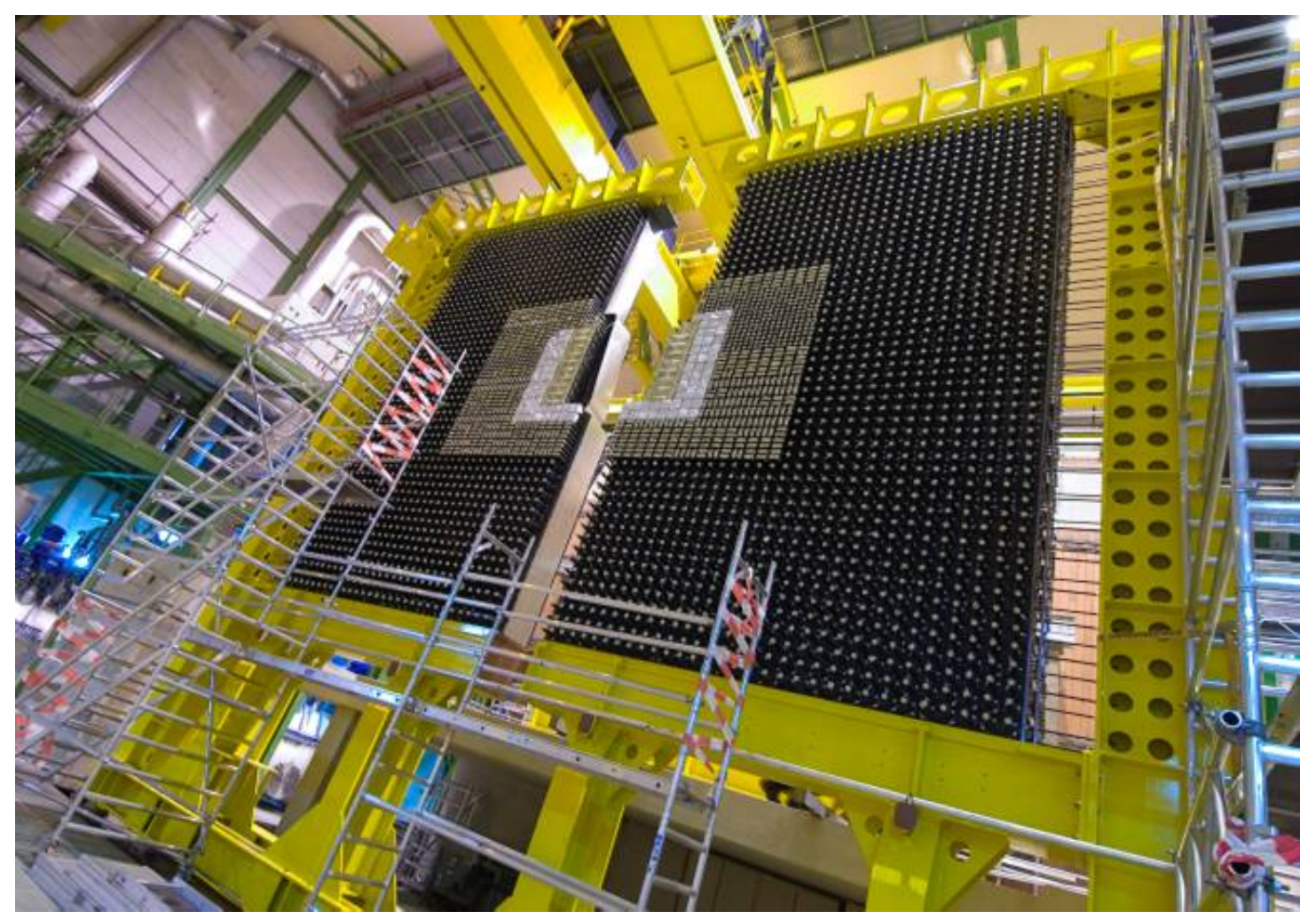




\section{Muon identification}

\section{Muon ID efficiency (\%) vs $\mu-\pi$ DLL cut}

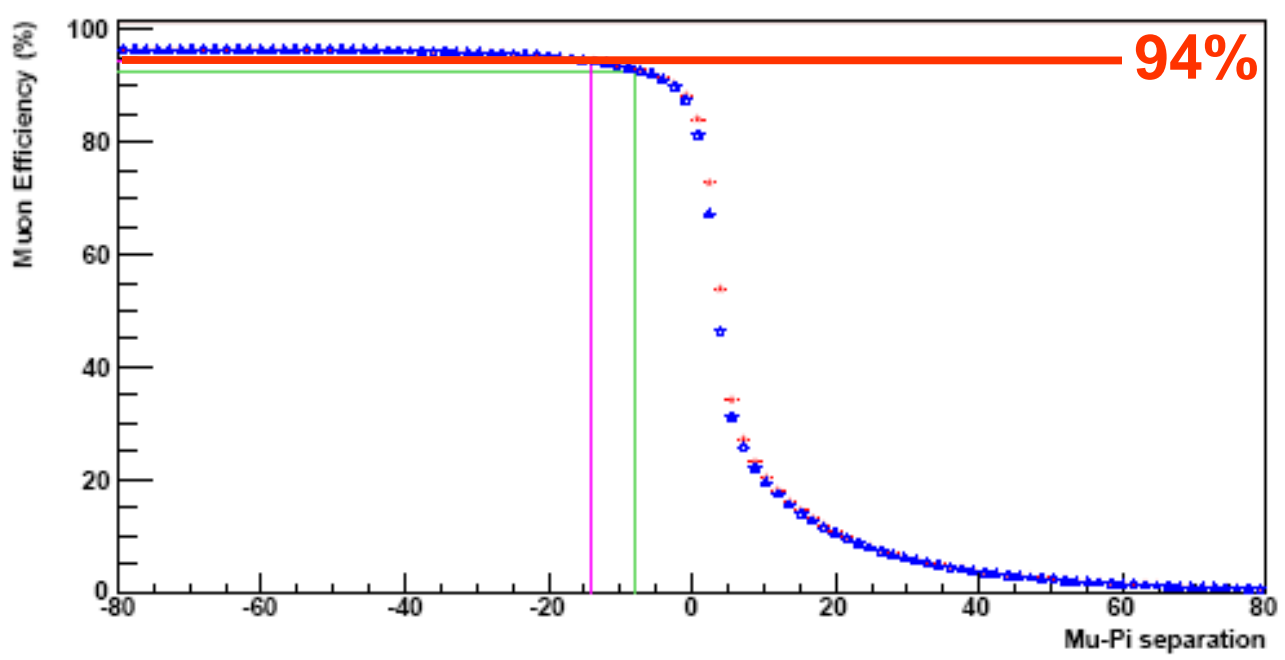

Pion mis ID efficiency (\%) vs $\mu-\pi$ DLL cut
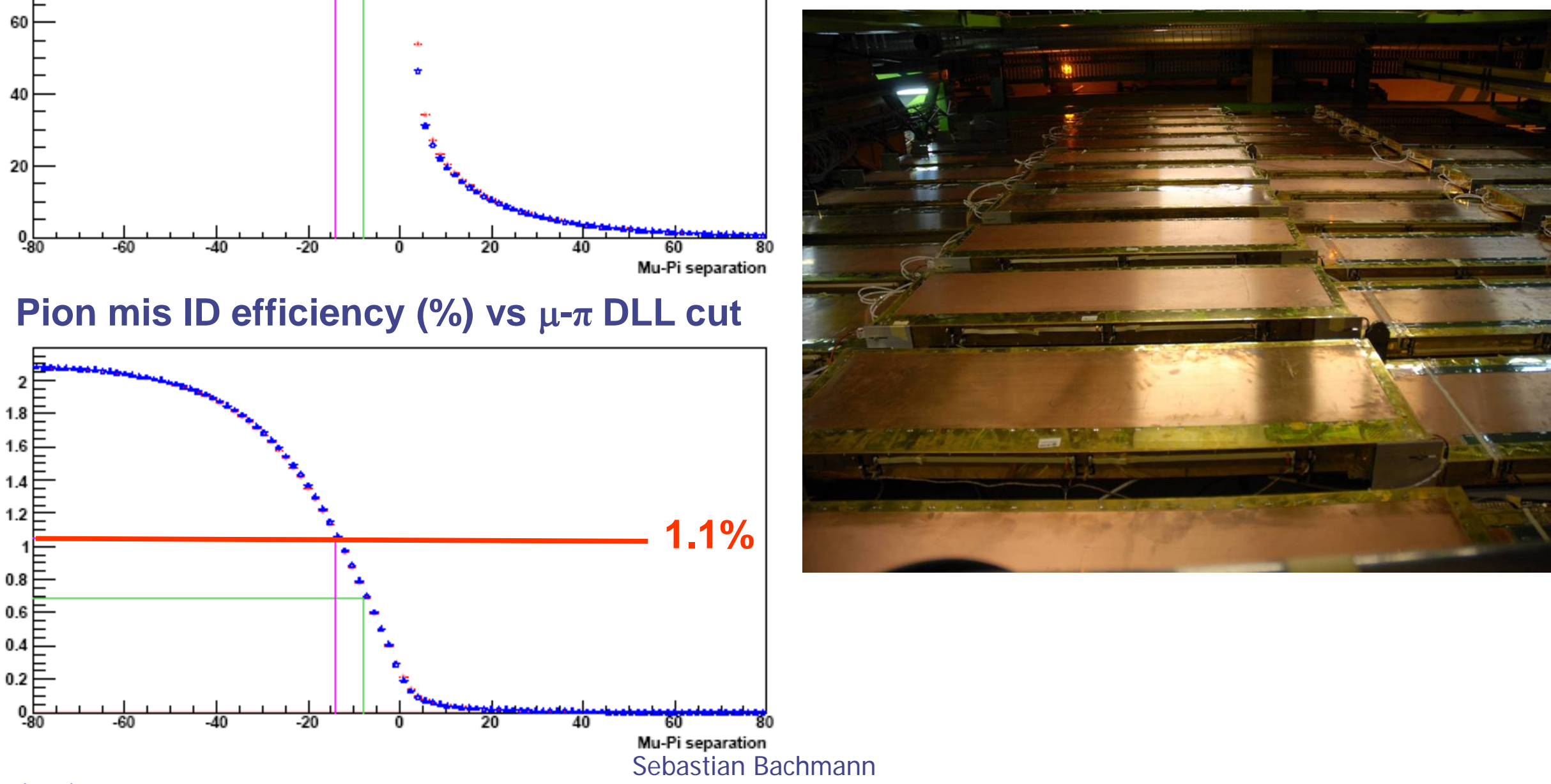
Trigger

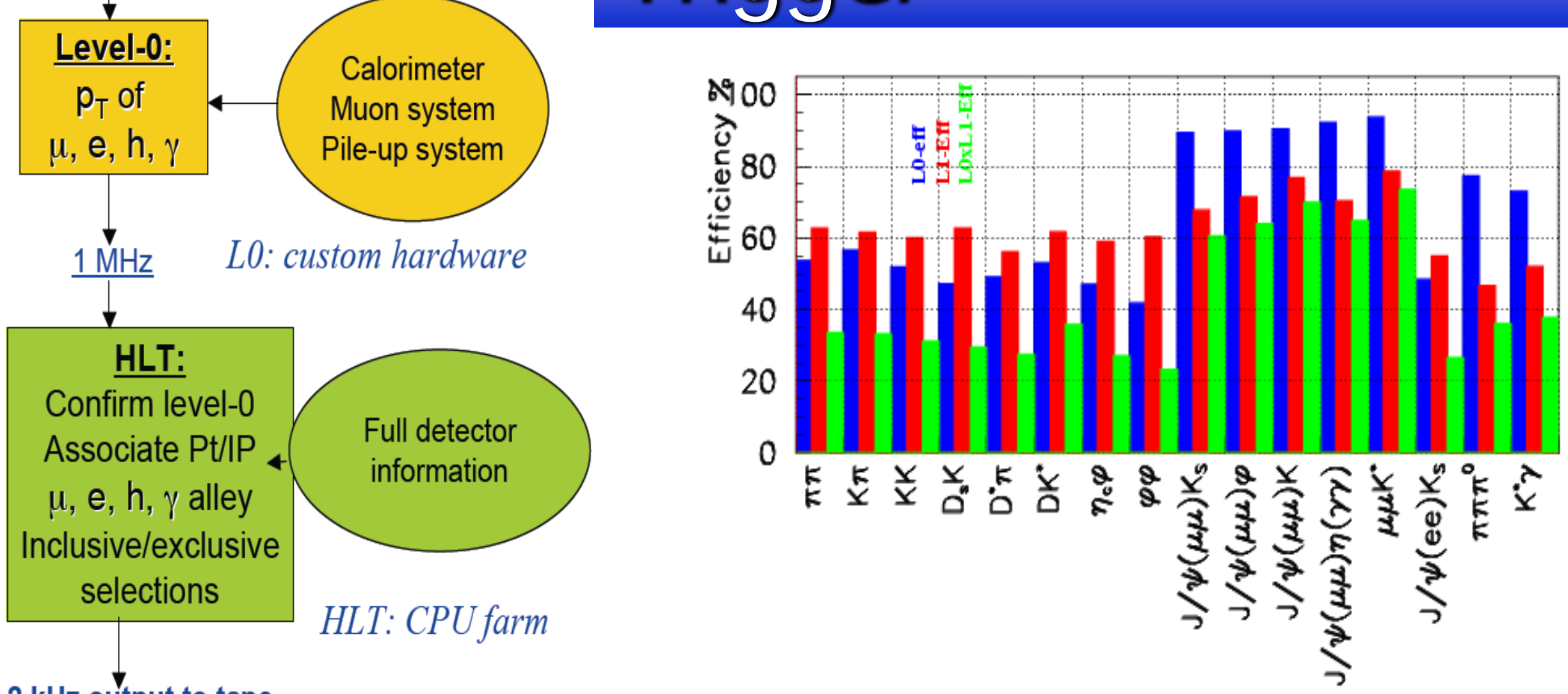

\section{HLT rate Event type}

$200 \mathrm{~Hz}$ Exclusive B candidates $600 \mathrm{~Hz}$ High mass di-muons $300 \mathrm{~Hz} \quad \mathrm{D}^{\star}$ candidates $900 \mathrm{~Hz}$ Inclusive b (e.g. $b \rightarrow \mu$ )
Calibration

Tagging

Tracking $\quad J / \psi, b \rightarrow J / \psi X$ (unbiased)

PID Charm (mixing \& CPV)

Trigger
Physics

B (core program)

B (data mining) 


\section{Flavor tagging}

\section{$>$ Opposite side}

- Charge of the kaon in the $b \rightarrow c \rightarrow s$ chain

- Charge of the lepton in semi-leptonic decays

- Charge of accompanying $b$ jet

\section{$>$ Same side}

- Charge of the $\mathrm{K}$ accompanying $\mathrm{B}_{\mathrm{s}}$

- Charge of the $\pi$ from $B^{* *} \rightarrow B^{*} \pi$

Tag

Opposite $\mu$

Opposite e

Opposite K

Opposite $\mathrm{Q}_{v t s}$

Same side $\pi\left(B^{0}\right)$

Same side $\mathrm{K}\left(\mathrm{B}_{\mathrm{s}}\right)$

Combined $\left(\mathrm{B}^{0}\right)$

Combined $\left(\mathrm{B}_{\mathrm{s}}\right)$

$$
\varepsilon D^{2}=\varepsilon(1-2 w)^{2}
$$

$0.7 \%-1.8 \%$

$0.4 \%-0.6 \%$

$1.6 \%-2.4 \%$

$0.9 \%-1.3 \%$

$0.8 \%-1.0 \%$

$2.7 \%-3.3 \%$

$4 \%-5 \%$

$7 \%-9 \%$

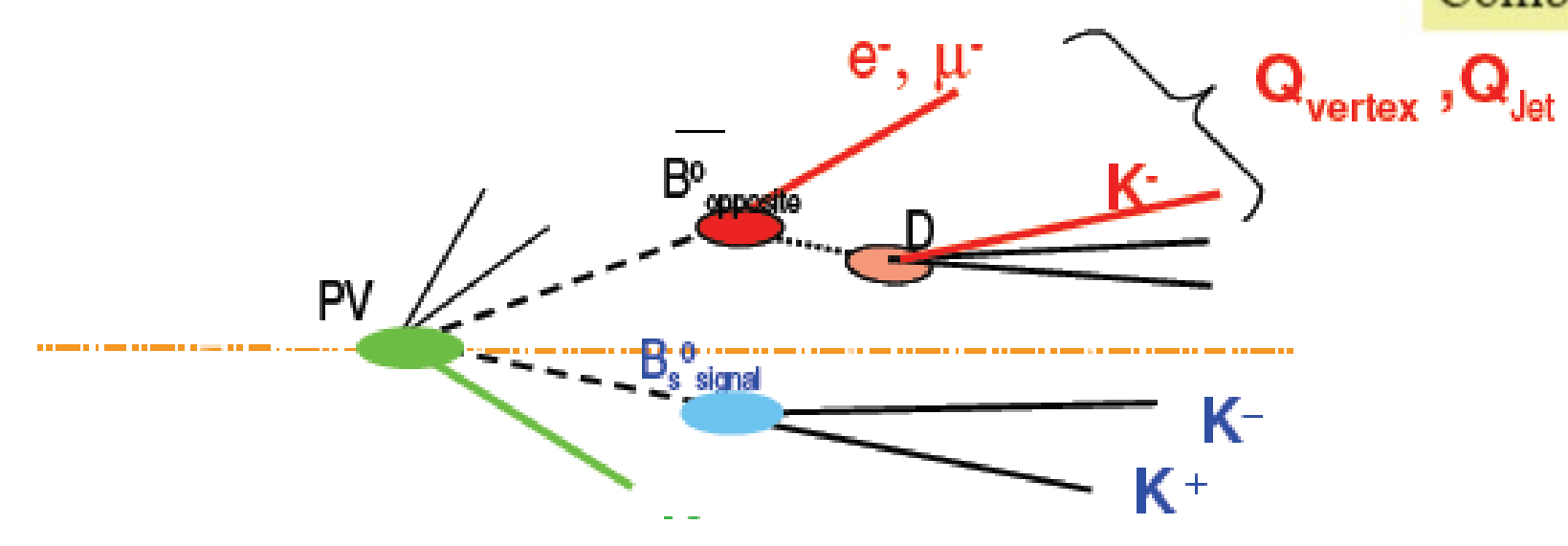




\section{$\Delta \mathrm{m}_{\mathrm{s}}$ from $\mathrm{B}_{\mathrm{s}} \rightarrow \mathrm{D}_{\mathrm{s}}^{-}\left(\mathrm{K}^{+} \mathrm{K}^{-} \pi^{-}\right) \mathrm{K}^{+}$}

$B_{s}^{0} \rightarrow D_{s}^{-} \pi^{+}$

-has a large branching fraction of (3.4 0.7) $\times 10^{-3}$.

- is flavor specific.

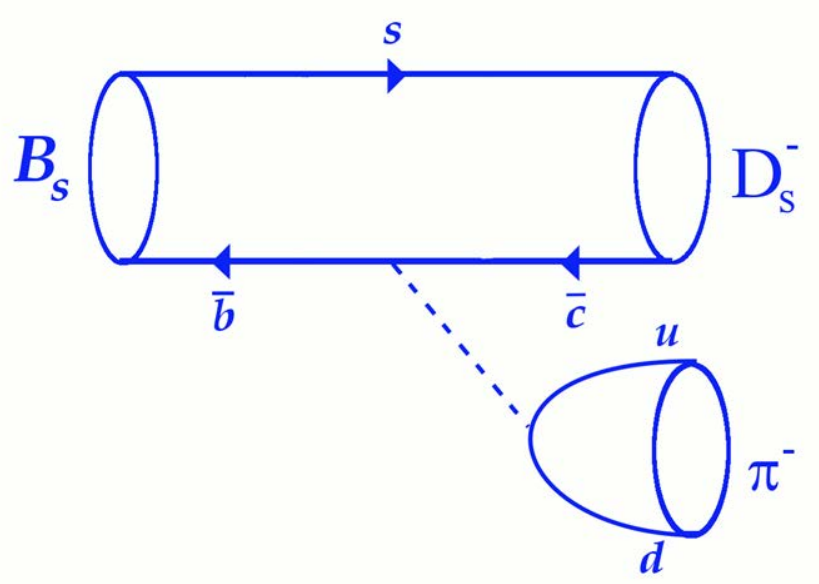

Total efficiency: $\varepsilon_{\text {tot }}=0.39 \%$

Signal yield: $\quad 140 \mathrm{k} \quad 0.67 \mathrm{k}$ (stat.) $\pm 40 \mathrm{k}$ (syst.)

(assuming 1 year of nominal running,

i.e. $\left.2 \mathrm{fb}^{-1}\right)$

$\mathrm{B} / \mathrm{S}$ at $90 \% \mathrm{CL}:[0.014,0.05]$ (bb combinatorial)

$[0.08,0.4] \quad$ (bb specific) 


\section{Sensitivity to $\Delta \mathrm{m}_{\mathrm{s}}$}

$>$ Plot made for 1 year of data (80k selected events, LHCb) for $\Delta \mathrm{m}_{\mathrm{s}}=20 \mathrm{ps}^{-1}$

> Control of mistag rate, resolution, background and acceptance important

$>$ Expected sensitivity for $2 \mathrm{fb}-1$ (i.e. year of data) $\sigma\left(\Delta \mathrm{m}_{\mathrm{s}}\right)=0.007 \mathrm{ps}^{-1}$

CDF: $\Delta \mathrm{m}_{\mathrm{s}}=(17.8 \pm 0.1) \mathrm{ps}^{-1}$

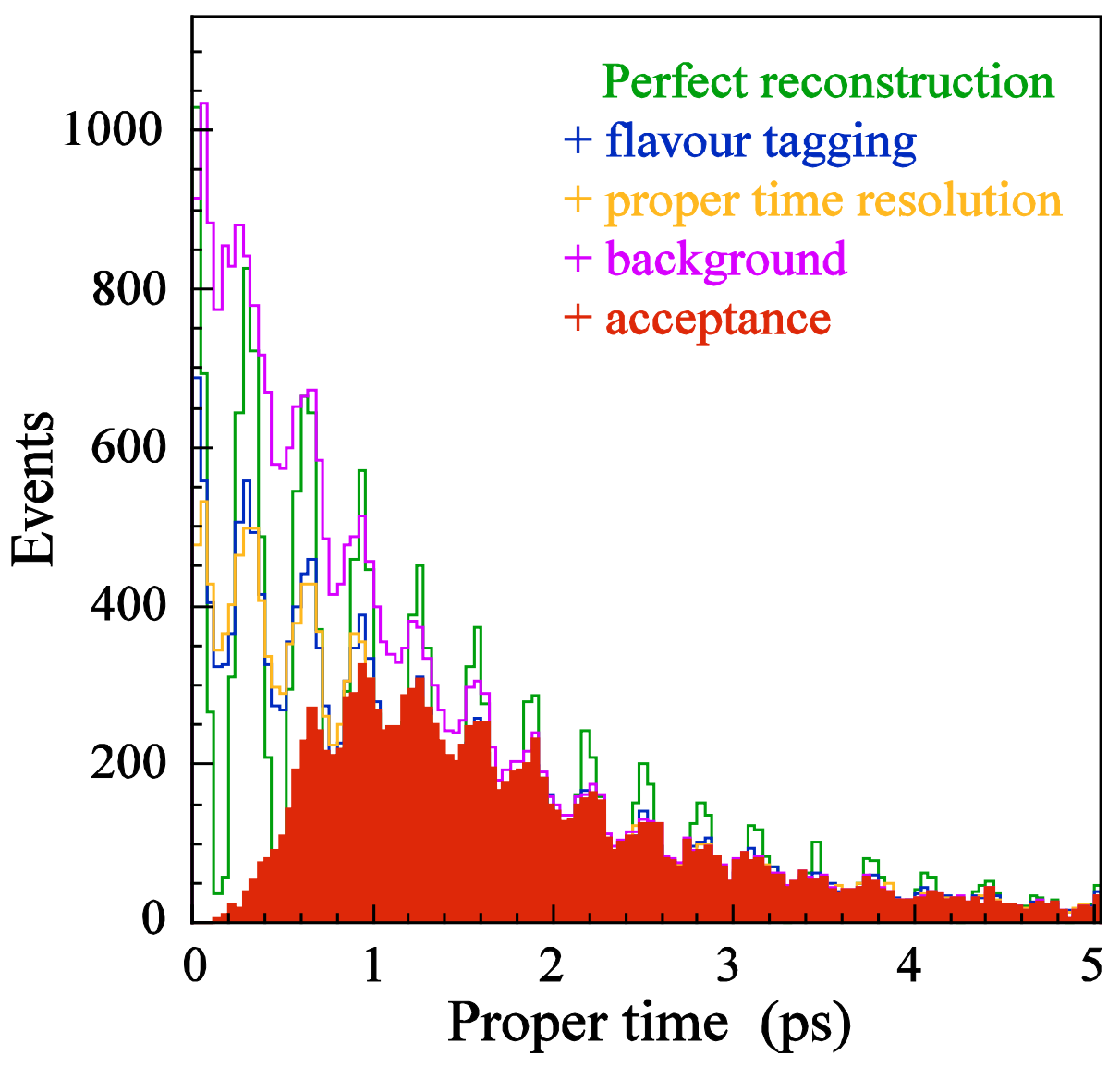




\section{Sensitivity for New Physics}

Model independent parametrization for New Physics:

\section{$M_{12}=\left(1+h_{s} \mathrm{e}\right.$}

$$
\begin{gathered}
\Delta m_{s}=\Delta m_{s}^{S M}\left|1+h_{s} \exp (2 i) \sigma_{s}\right| \\
\phi_{s}=\phi_{s}^{S M}+\arg \left(1+h_{s} \exp \left(2 i \sigma_{s}\right)\right)
\end{gathered}
$$

$\Delta \Gamma_{s}=\Delta \Gamma_{s}^{S M} \cos ^{2}\left(\arg \left(1+h_{s} \exp \left(2 i \sigma_{s}\right)\right)\right)$
In april 2006, including first CDF measurement of $\Delta \mathrm{m}_{\mathrm{s}}$

\section{from hep-ph/0604112}

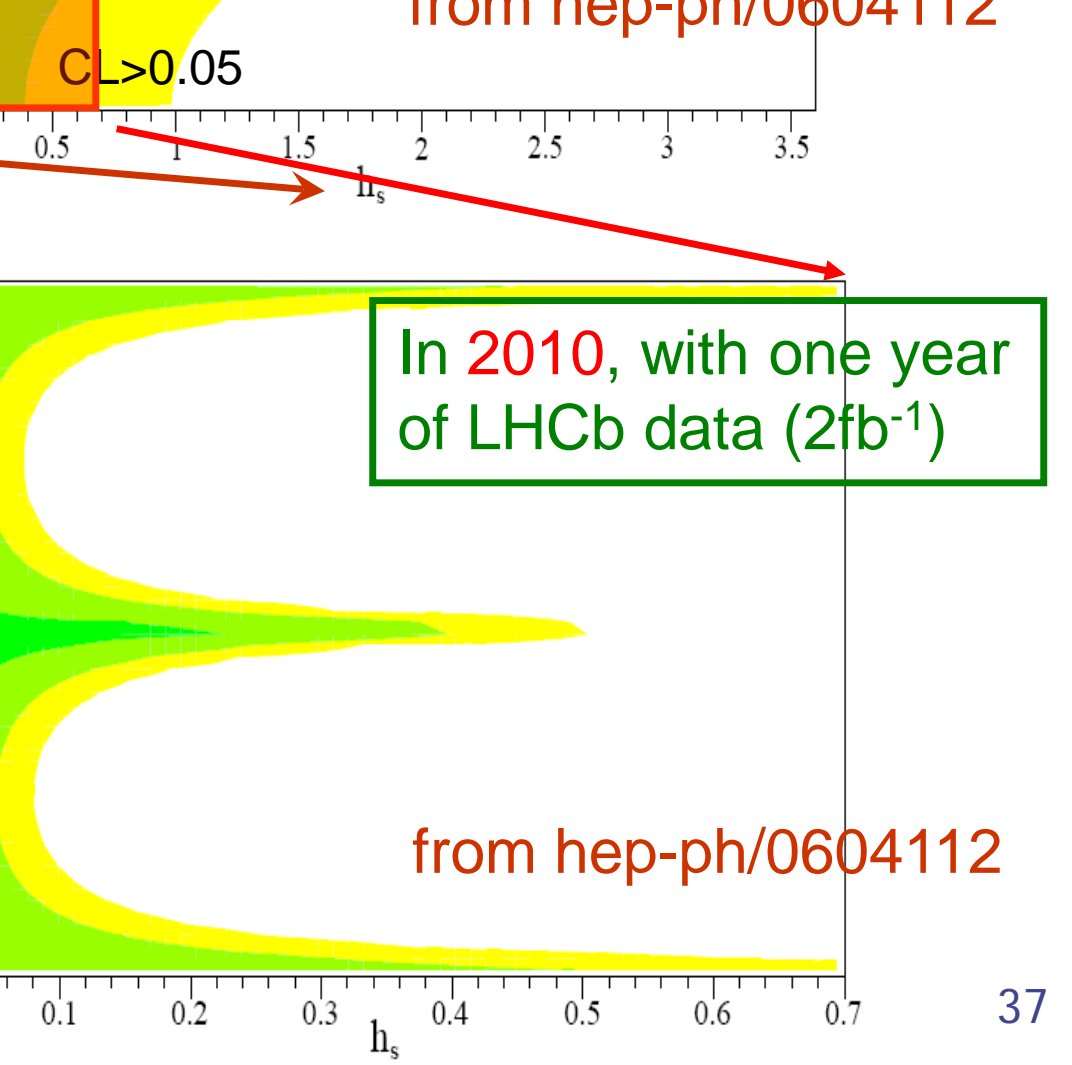




\section{$\mathrm{B}_{\mathrm{s}} \rightarrow \Phi\left(\mathrm{K}^{+} \mathrm{K}^{-}\right) \Phi\left(\mathrm{K}^{+} \mathrm{K}^{-}\right)$}

- FCNC (b $\rightarrow$ SSs)with SM prediction for CP-asymmetry < 1\%. see e.g. M.Raidal, PRL 89,231803(2002)

- Sizeable CP asymmetry is an unambiguous sign for NP.

- Like in $\mathrm{B}_{\mathrm{s}} \rightarrow \mathrm{J} / \Psi \phi$ a full angular analysis to extract CPasymmetry is needed.

- Experimentally demanding, as full hadronic trigger is needed.

Remark:

- In $\mathrm{SM} \mathrm{B} \mathrm{B}_{\mathrm{s}} \rightarrow \mathrm{J} / \psi \phi$ and $\mathrm{B}_{\mathrm{s}} \rightarrow \phi \phi$ measure both $\arg \left[V_{t b}{ }^{*} V_{t s}\right]$.

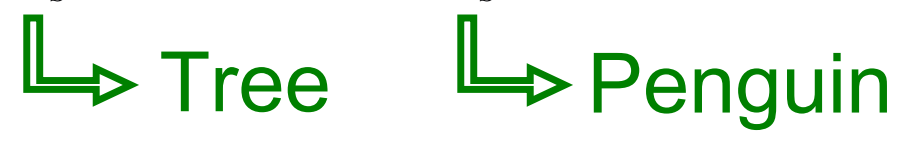

- Deviations point to physics beyond the SM.

- Belle/Babar have 2.7 $\sigma$ deviation when comparing tree and penguin decays of $\mathrm{B}_{\mathrm{d}}$ to CP-Eigenstate. 


\section{Event selection and sensitivity studies}

\section{Selection:}

$>$ Reconstruct only $\phi \rightarrow \mathrm{K}^{+} \mathrm{K}^{-}$.

$>$ Full detector simulation including trigger bias.

$>$ Reconstruction based on:

o RICH K ID

o pt and impact parameter of $\mathrm{K}^{ \pm}$ and $\phi$ candidates.

$o \mathrm{~B}_{\mathrm{s}}$ and $\phi$ invariant mass.

$o B_{s}$ and $\phi$ vertex quality.

Sensitivity to $\phi_{\mathrm{S}}$ studied by toy MC:

- proper time resolution of 42fs.

- proper time acceptance function.

- flat BG in $m_{B}$ and transversity angle.

- mistag dilution: $\varepsilon(1-2 \omega)=9.6 \%$

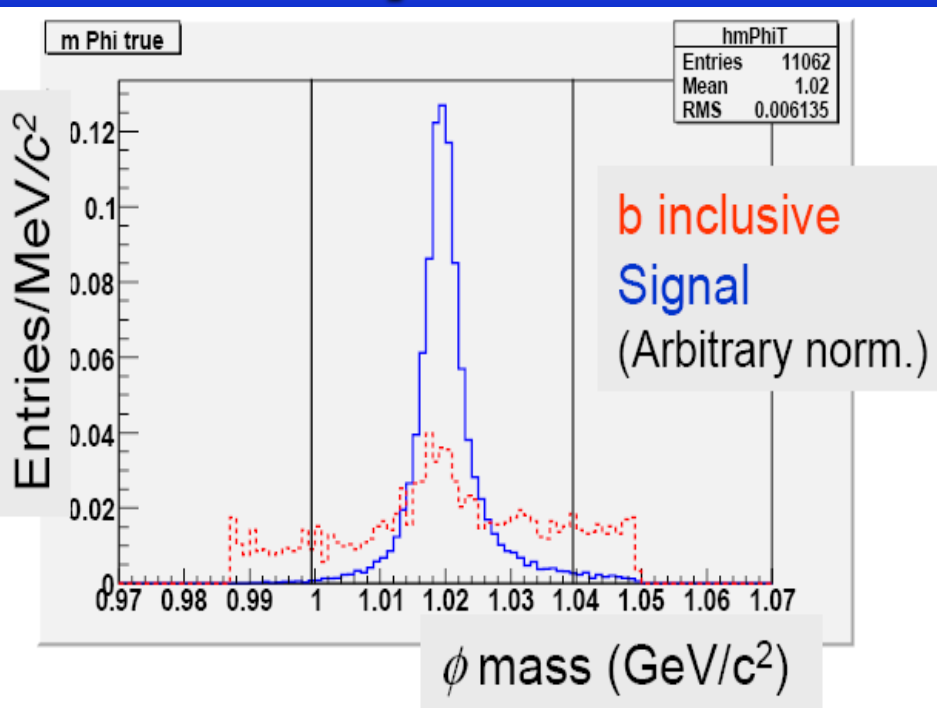

- exponential lifetime distribution for BG

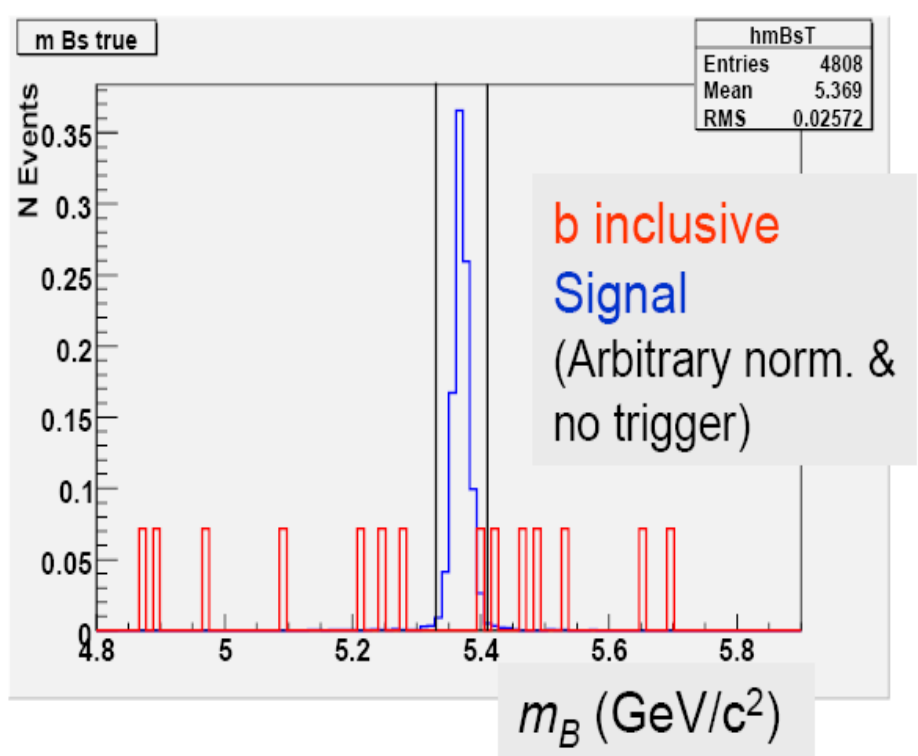




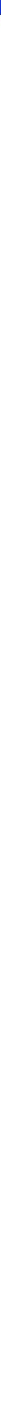

From G. Hiller [hep-ph/0308180] 


\section{2.) $\mathrm{B}^{0} \rightarrow \mathbb{K}^{* 0} \mu^{+} \mu^{-}$}

\section{SM processes contributing to decay:}
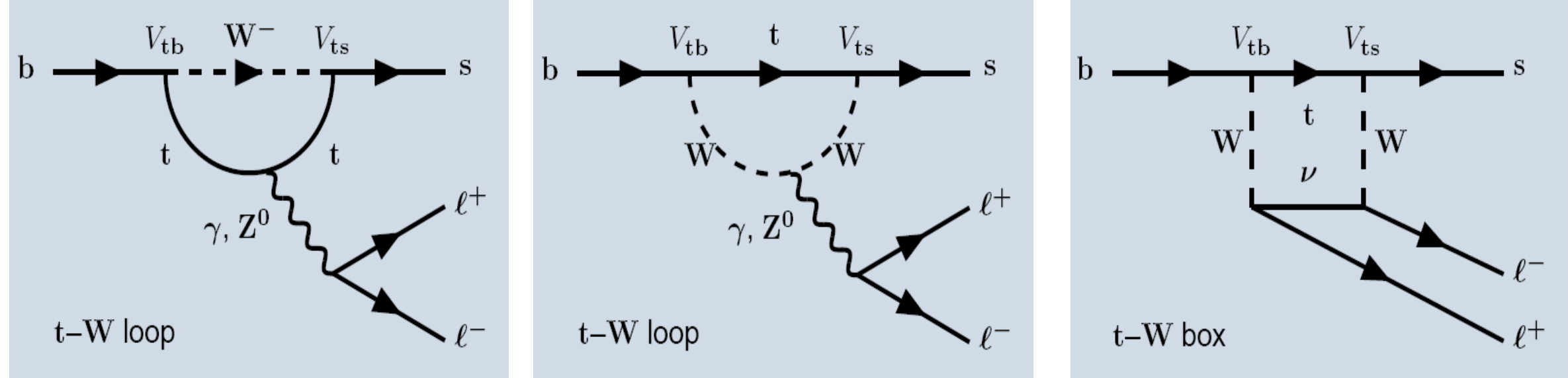

$$
\begin{aligned}
& \mathrm{BR}\left(\mathrm{B}^{0} \rightarrow 11 \mathrm{~s}\right)=4.5 \times 10^{-6} \\
& \mathrm{BR}\left(\mathrm{B}^{0} \rightarrow 11 \mathrm{~K}\right)=0.5 \times 10^{-6}
\end{aligned}
$$

Decay is very sensitive to extensions of SM, especially to models with right handed currents:

Analysis of angular distributions allow to extract this information about new Physics. 


\section{Observables in $\mathrm{B}^{0} \rightarrow \mathbb{K}^{* 0} \mu^{+} \mu^{-}$}

Two observables are of special interest, as they have small theoretical errors and are very sensitive to NP:

Forward-Backward Asymmetry in $\theta_{\underline{I}}$ :

$$
A_{F B}(s)=\frac{\int_{0}^{1} \frac{\mathrm{d}^{2} \Gamma}{\mathrm{d} s \mathrm{~d} \cos \theta} \mathrm{d} \cos \theta-\int_{-1}^{0} \frac{\mathrm{d}^{2} \Gamma}{\mathrm{d} s \mathrm{~d} \cos \theta} \mathrm{d} \cos \theta}{\int_{0}^{1} \frac{\mathrm{d}^{2} \Gamma}{\mathrm{d} s \mathrm{~d} \cos \theta} \mathrm{d} \cos \theta+\int_{-1}^{0} \frac{\mathrm{d}^{2} \Gamma}{\mathrm{d} s \mathrm{~d} \cos \theta} \mathrm{d} \cos \theta}
$$

Transverse Asymmetry:

(asymmetry in the spin amplitude of the $\mathrm{K}^{\star}$ )

$$
A_{T}^{(2)}(s)=\frac{\left|A_{\perp}\right|^{2}-\left|A_{\|}\right|^{2}}{\left|A_{\perp}\right|^{2}+\left|A_{\|}\right|^{2}} .
$$




\section{$\mathrm{A}_{\mathrm{FB}}$ and $\mathrm{A}_{\mathrm{T}}(2)$}

AFB(s) in SM and different SUSY models:

SUSY I = SUGRA

SUSY II = MIA MSSM

(from Phys.Rev.D61 (2000) 074024)

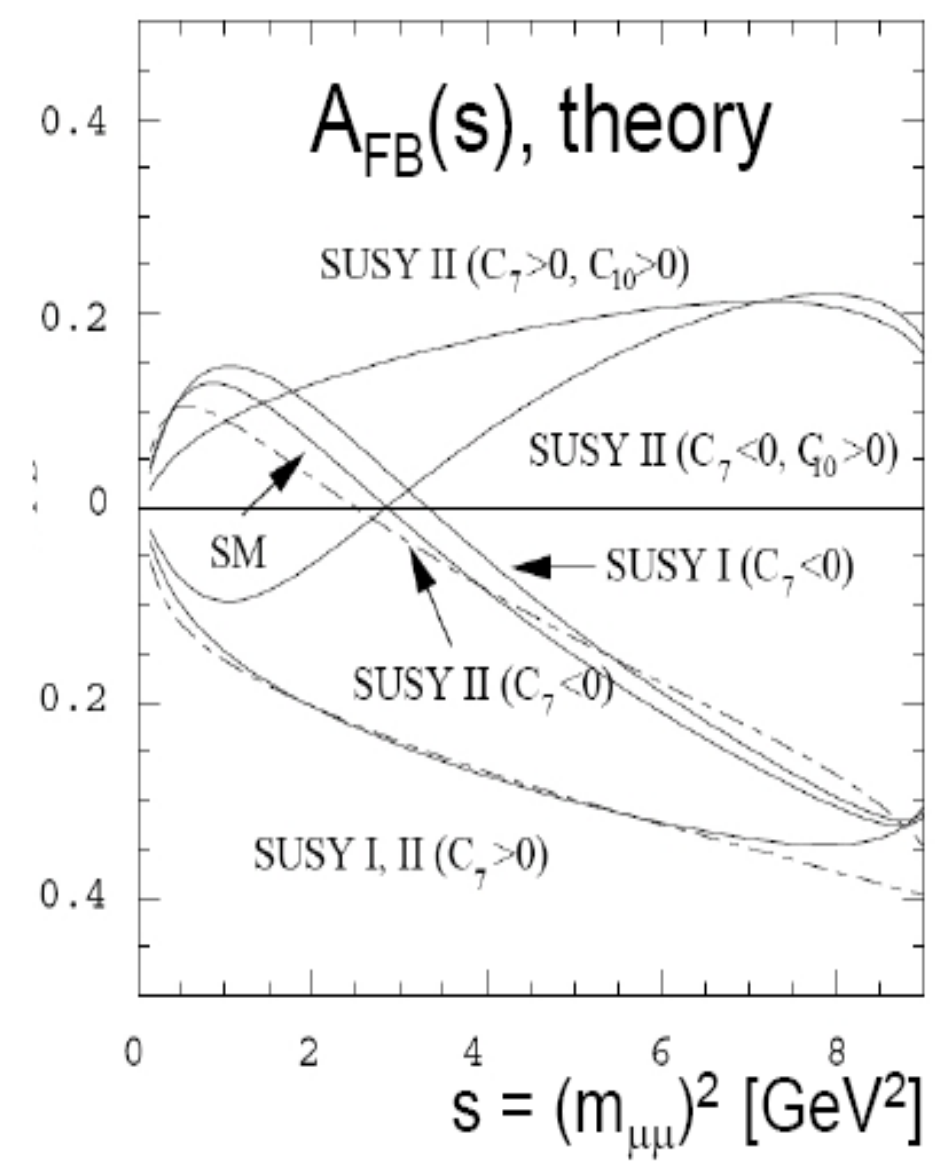

Non-MFV MSSM with $\tan (\beta)=5$

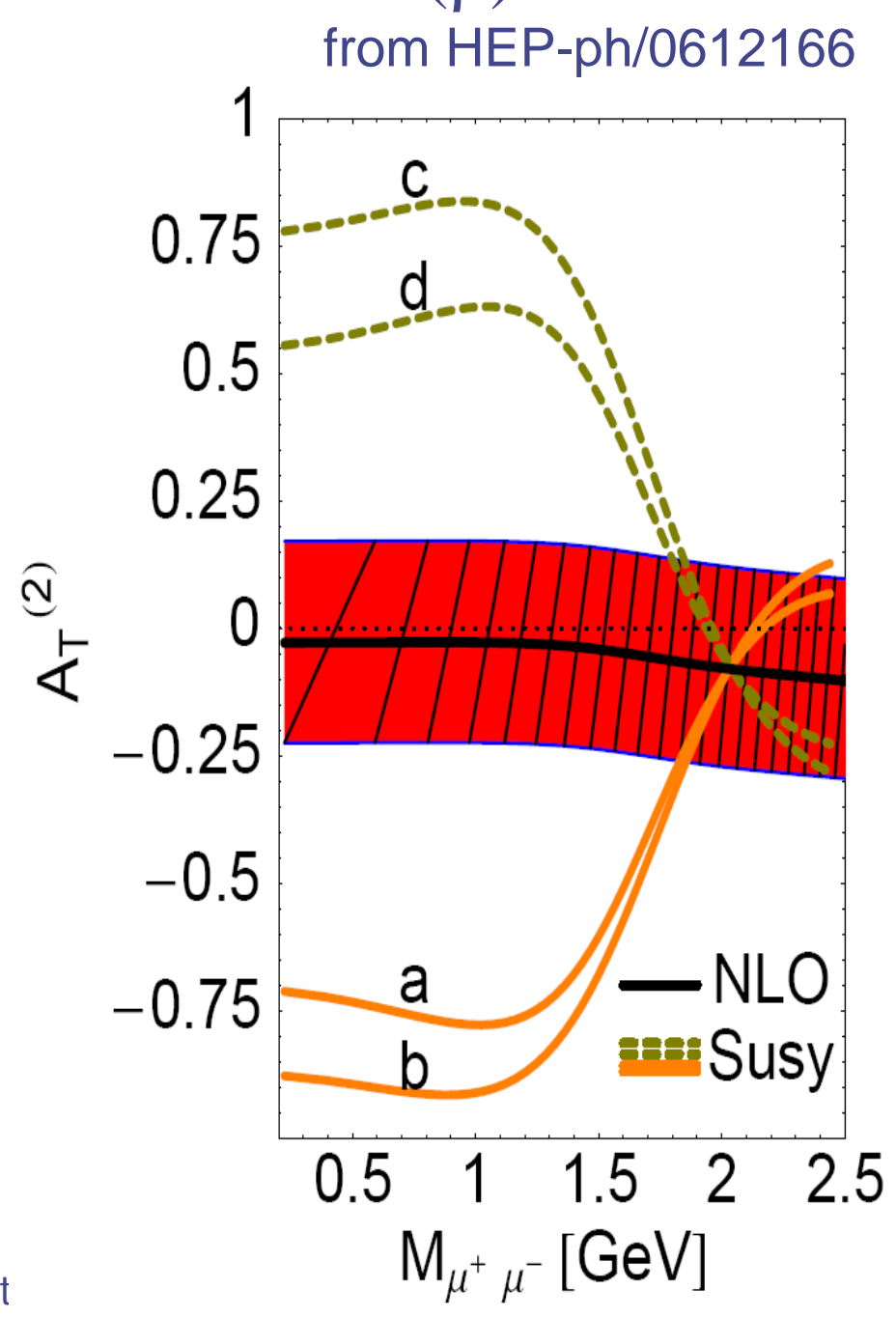




\section{Selection of events: $\mathrm{B}^{0} \rightarrow \mathbb{K}^{*} \mu^{+} \mu^{-}$}

Expected yield for $2 \mathrm{fb}^{-1}$ :

$7200 \pm 180$ (stat.) \pm 2200 (BR)

Estimate for Background:

\section{MC sample}

$\mathrm{Bd}, \mathrm{u} \rightarrow \mathrm{s} \mu \mu\left(\right.$ no $\left.\mathrm{K}^{*}\right)$

$\mathrm{b} \rightarrow \mu, \mathrm{b} \rightarrow \mu$

$\mathrm{b} \rightarrow \mu, \mathrm{c} \rightarrow \mu$

Total
No. of events per $2 \mathbf{f b}^{-1}$

$9 \pm 3$
$1050 \pm 250$
690180
$1770 \quad 310$

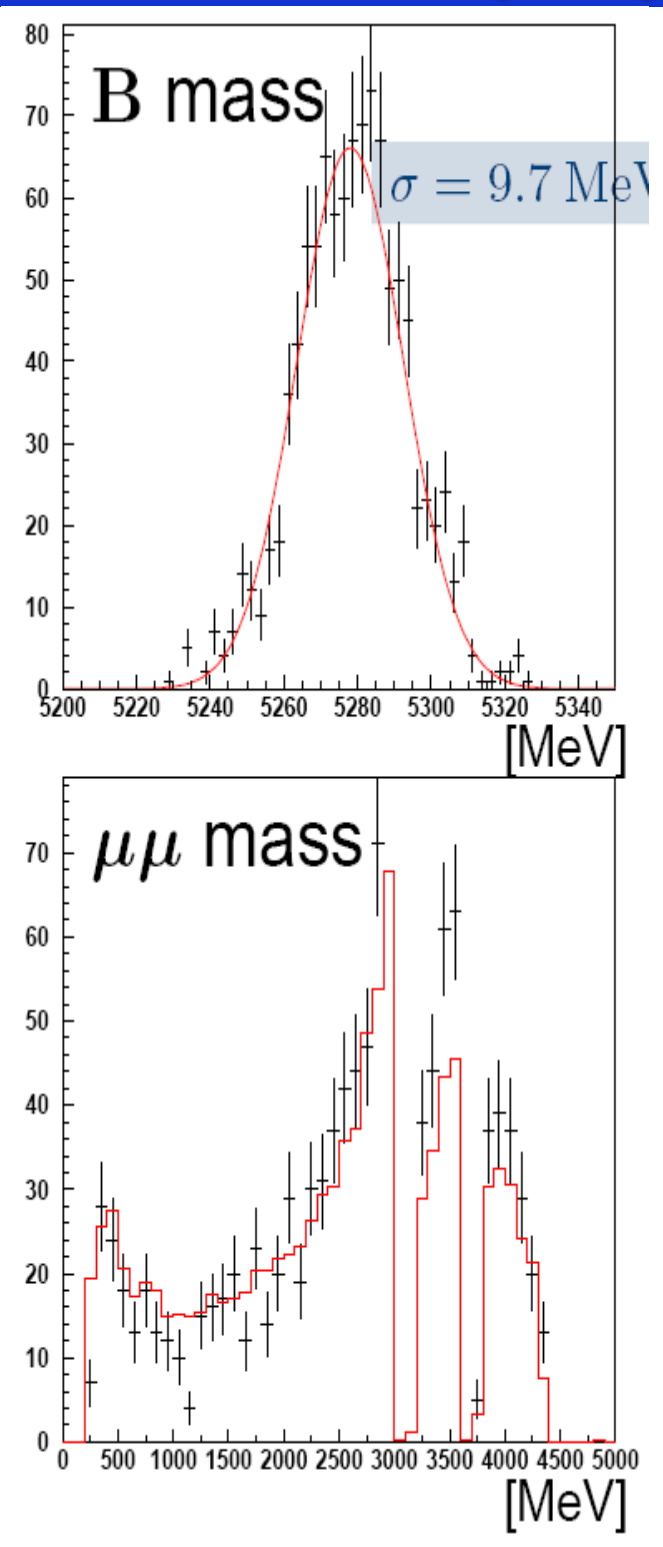




\section{$A_{F B}$ and $A_{T}(2)$ from $B^{0} \rightarrow K^{* 0} \mu \mu$}

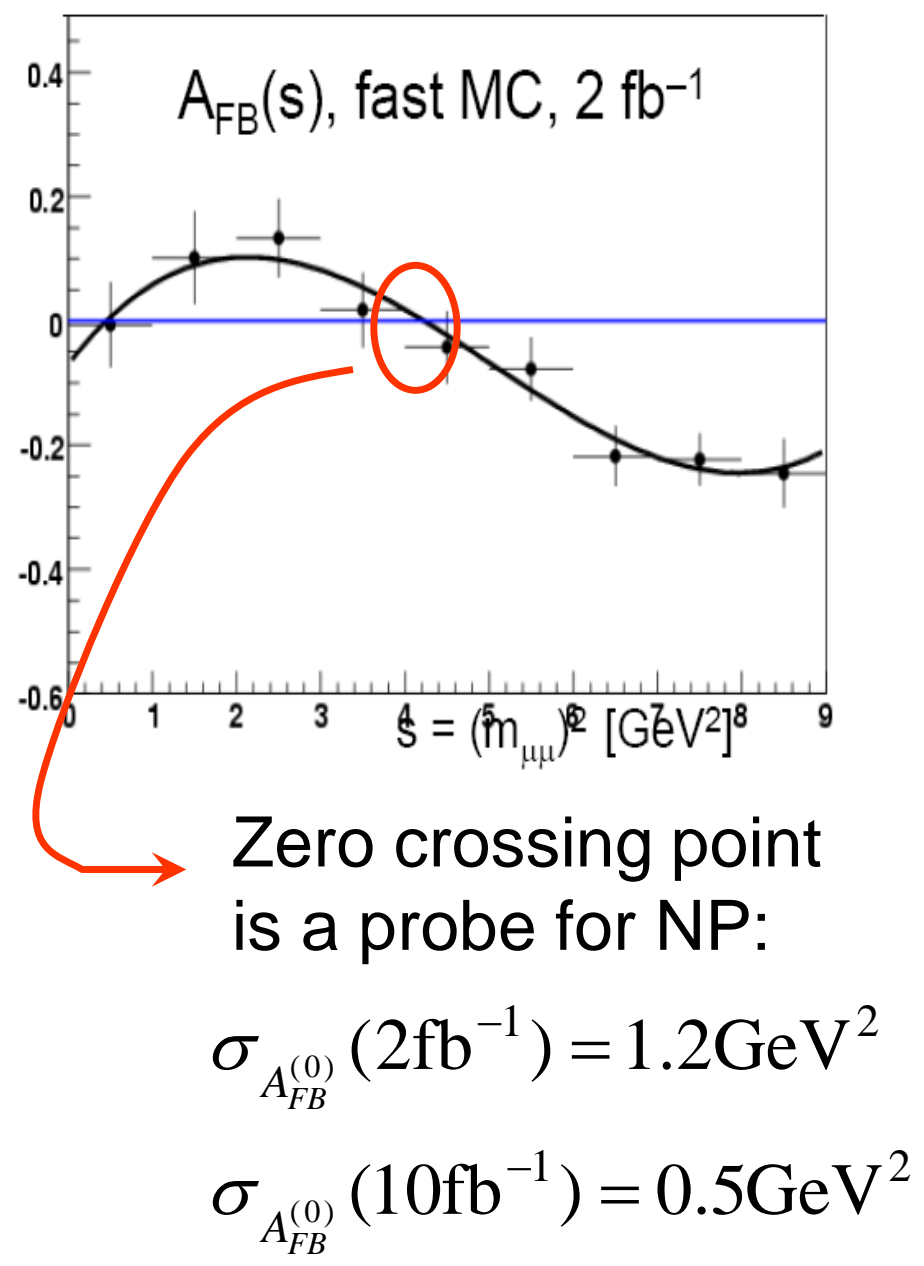

1 year of data taking, errors expected to be limited by statistics

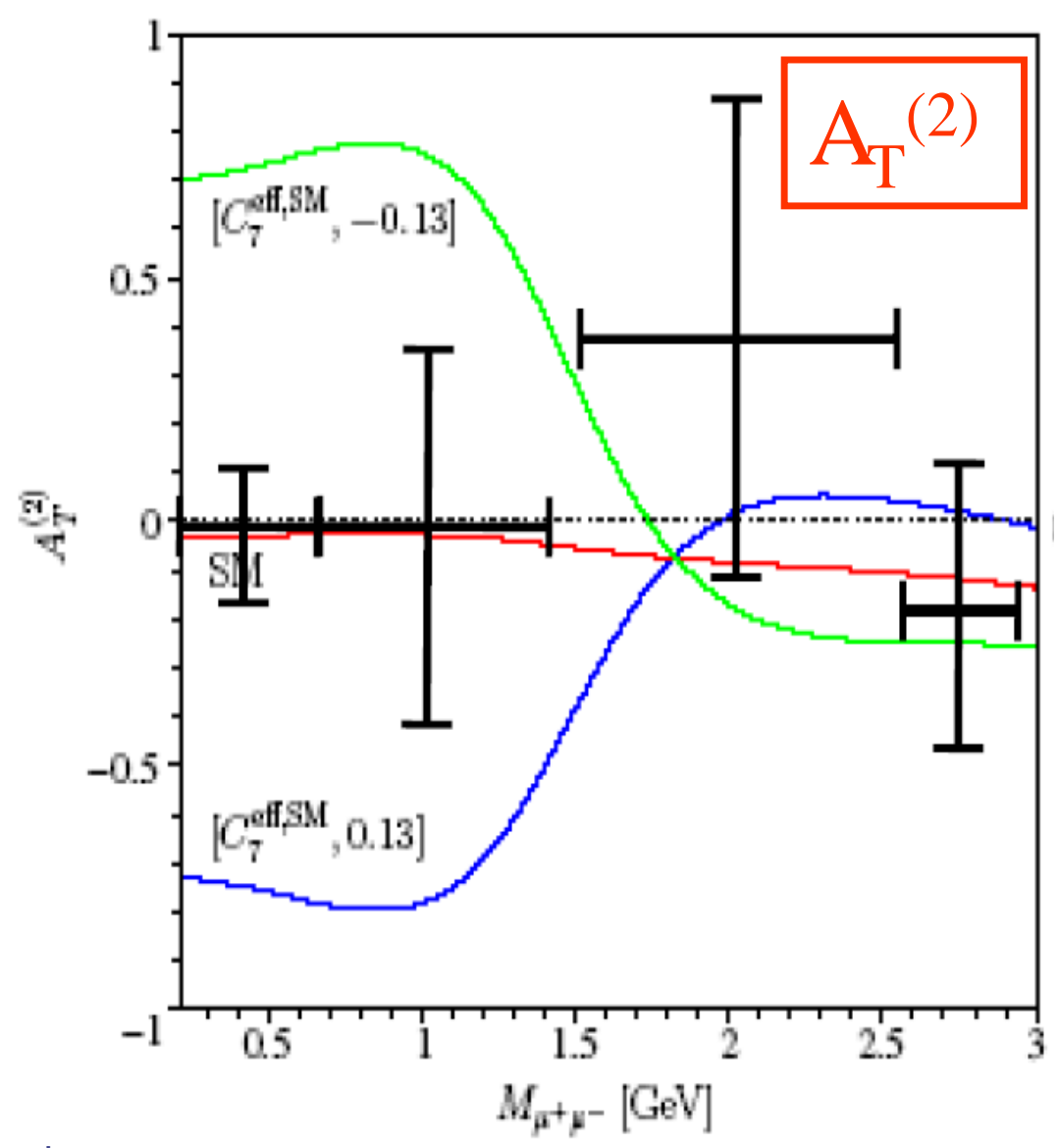

